\title{
СОЦИАЛЬНО-ЭКОНОМИЧЕСКИЕ ФАКТОРЫ И ПОСЛЕДСТВИЯ ИЗБЫТОЧНОГО ВЕСА
}

\author{
МАРИНА КОЛОСНИЦЫНА, ОЛЬГА КУЛИКОВА
}

\begin{abstract}
Растущий во всем мире избыточный вес населения - одна из важнейших угроз общественному здоровью. В последние десятилетия этот феномен активно исследуется представителями разных наук. В России сочиально-экономические факторы и последствия избыточного веса пока мало изучены. В статье представлен обзор литературы, посвященной причинам избыточного веса и издержкам, которые несут отдельные индивиды, работодатели, государство. На базе микроданных Российского мониторинга экономического положения и здоровья населения за 2012-2016 г2. проводится эмпирический анализ динамики распространения ожирения и предожирения в стране, изучается связь избыточного веса с демографическими и социально-экономическими факторами, с положением работника на рынке труда, расходами на лечение.
\end{abstract}

Результаты анализа подтверждают связь склонности к избыточному весу с возрастом и гендерной принадлежностью человека, с факторами здорового образа жизни. Высшее и среднее специальное образование коррелирует с низким весом только среди женщин. Для мужчин, напротив, более высокий уровень образования ассочиируется с высокими показателями предожирения и ожирения. Связи избыточного веса и субъективного благосостояния не выявлено.

Оиенки связи избыточного веса и положения работников на рынке труда, проведенные регрессионными методами, показали, что индекс массы тела (ИМТ) значимо коррелирует как с вероятностью занятости, так и с заработной платой (для мужчин). Однако в обоих случаях эта связь оказывается положительной для низких и средних значений ИМТ, и лишь в случаях тяжелого ожирения наблюдается отрицательная зависимость.

Увеличение веса ассоциируется с ростом числа хронических заболеваний, ухудшением самооценки здоровья, растущей вероятностью инвалидности. Полные люди в среднем чаще попадают в больнииу, покупают лекарства и тратят на них больше денег по сравнению с людьми с нормальным весом. Однако эконометрический анализ подтвердил положительную связь веса и медицинских расходов только для женщин и для людей, страдающих предожирением, при прочих равных условиях.

Ключевые слова: ожирение, избыточный вес, индекс массы тела, последствия ожирения, РМЭЗ НИУ ВШЭ, Россия.

\section{ВВЕДЕНИЕ}

За последние десятилетия во всем мире достигнуты значительные успехи в улучшении здоровья населения, что отражается растущими показателями ожидаемой продолжительности жизни, в том числе - здоровой жизни. Во многом это результат не столько развития и распространения новых медицинских технологий, сколько изменения образа жизни людей. Экономический рост, рост доходов и образования населения, сокращение бедности дают возможности для лучших условий проживания, питания, проведения досуга.

МАРИНА ГРИГОРЬЕВНА КОЛОСНИЦЫНА (mkolosnitsyna@hse.ru), НАЦИОНАЛЬНЫЙ ИССЛЕДОВАТЕЛЬСКИЙ УНИВЕРСИТЕТ «ВЫСШАЯ ШКОЛА ЭКОНОМИКИ», РОССИЯ.

ОЛЬГА АНАТОЛЬЕВНА КУЛИКОВА, НАЦИОНАЛЬНЫЙ ИССЛЕДОВАТЕЛЬСКИЙ УНИВЕРСИТЕТ «ВЫСШАЯ ШКОЛА ЭКОНОМИКИ», РосСия.

СТАТЬЯ ПОДГОТОВЛЕНА В РАМКАХ ПРОЕКТА «ВКЛАД ОТДЕЛЬНЫХ ФАКТОРОВ В ФОРМИРОВАНИЕ ЗДОРОВЬЯ НАСЕЛЕНИЯ» ТЕМАТИЧЕСКОГО ПЛАНА ПРИКЛАДНЫХ ИССЛЕДОВАНИЙ НИУ ВШЭ ЗА 2018 Г. (ТЗ-154).

СТАТЬЯ ПОСТУПИЛА В РЕДАКЦИЮ В СЕНТЯБРЕ 2018 Г. 
Улучшаются условия труда, транспортные возможности - все это позитивно отражается на общественном здоровье. Многие страны мира за последние годы существенно продвинулись в области борьбы с курением и избыточным потреблением алкоголя, что также вносит значительный вклад в сокращение заболеваемости и смертности, в том числе от внешних причин. Однако рост благосостояния и изменение образа жизни людей в современном мире породили новую проблему, признанную сегодня в качестве одного из главных вызовов общественному здоровью. Речь идет о растущем ожирении, которое уже официально называют пандемией XXI века. Впервые в истории человечества на рубеже XX-XXI веков число людей с избыточным весом, которые потребляют пищи больше, чем им нужно, сравнялось с числом страдающих от недоедания и недостатка веса: и тех, и других было около 1 млрд [Gardner, Halweil 2000]. За прошедшие с тех пор годы число людей с избыточным весом практически удвоилось и составило в 2016 г. 1,9 млрд человек или $39 \%$ населения мира ${ }^{1}$.

Растущий вес (как и рост) населения наблюдался в развитых странах еще с XVIII века, однако считался позитивным явлением, поскольку сопровождался улучшением здоровья и увеличением продолжительности жизни. Нобелевский лауреат, специалист по экономической истории Роберт Фогель предложил «теорию технофизической эволюции»: он показал, что в течение 300 лет физиология человека изменялась под воздействием прогресса технологий, в результате чего размеры его тела увеличились в среднем в полтора раза и возросла сопротивляемость внешним воздействиям, а потому снизилась смертность [Fogel, Costa 1997]. 1980-е годы оказались переломным десятилетием, когда темпы роста среднего индекса массы тела (ИМТ) стали вдвое и даже втрое более быстрыми, чем в предшествующее столетие [Sassi 2010: 18]. Если в 1970-80-е годы в большинстве развитых стран уровень ожирения был в пределах 5-15\%, а для многих развивающихся стран такого явления просто не существовало, то сегодня и богатые страны, и страны со средними доходами демонстрируют показатели 25-35\% (рисунок 1). Страны Ближнего Востока вплотную приближаются к США - признанному лидеру ожирения (36,2\% в 2016 г.), быстро «полнеют» Бразилия, Мексика, Новая Зеландия и Южная Африка. Франция, Финляндия и Норвегия, хотя и отстают, но также превысили 20 -процентный порог $(21,6 \%, 22,2 \%, 23,1 \%$ соответственно). В последние десять лет отмечается ускоренный рост ожирения в Индии, Китае и Японии. Россия демонстрирует аналогичную тенденцию: «средний» показатель ожирения в 2016 г. - 23,1\%, при этом темпы его прироста в 2010-2016 гг. увеличились.

Одновременно происходит рост доли населения в группе «предожирения», так что во многих развитых странах избыточным весом (ожирением и предожирением) страдает уже более половины взрослых людей.

Избыточный вес, прежде всего, ассоциируется с многочисленными болезнями, повышая риски развития сердечно-сосудистых заболеваний, диабета II типа, гипертонии, заболеваний суставов, некоторых видов злокачественных новообразований. Влияние ожирения на здоровье и медицинские расходы значительнее, чем последствия злоупотребления алкоголем и курения [Sturm 2002]. Специалисты ВОЗ констатируют, что

\footnotetext{
${ }^{1}$ URL: http://www.who.int/en/news-room/fact-sheets/detail/obesity-and-overweight (дата обращения: 19.08.2018). 
сегодня в мире больше людей умирает от болезней, связанных с ожирением, чем от недоедания, - 2,8 млн человек ежегодно ${ }^{2}$. Растут расходы национальных систем здравоохранения, а также косвенные затраты: сокращаются продуктивность работников и в целом экономический рост. Издержки ожирения имеют, таким образом, не только частное, но и общественное значение.

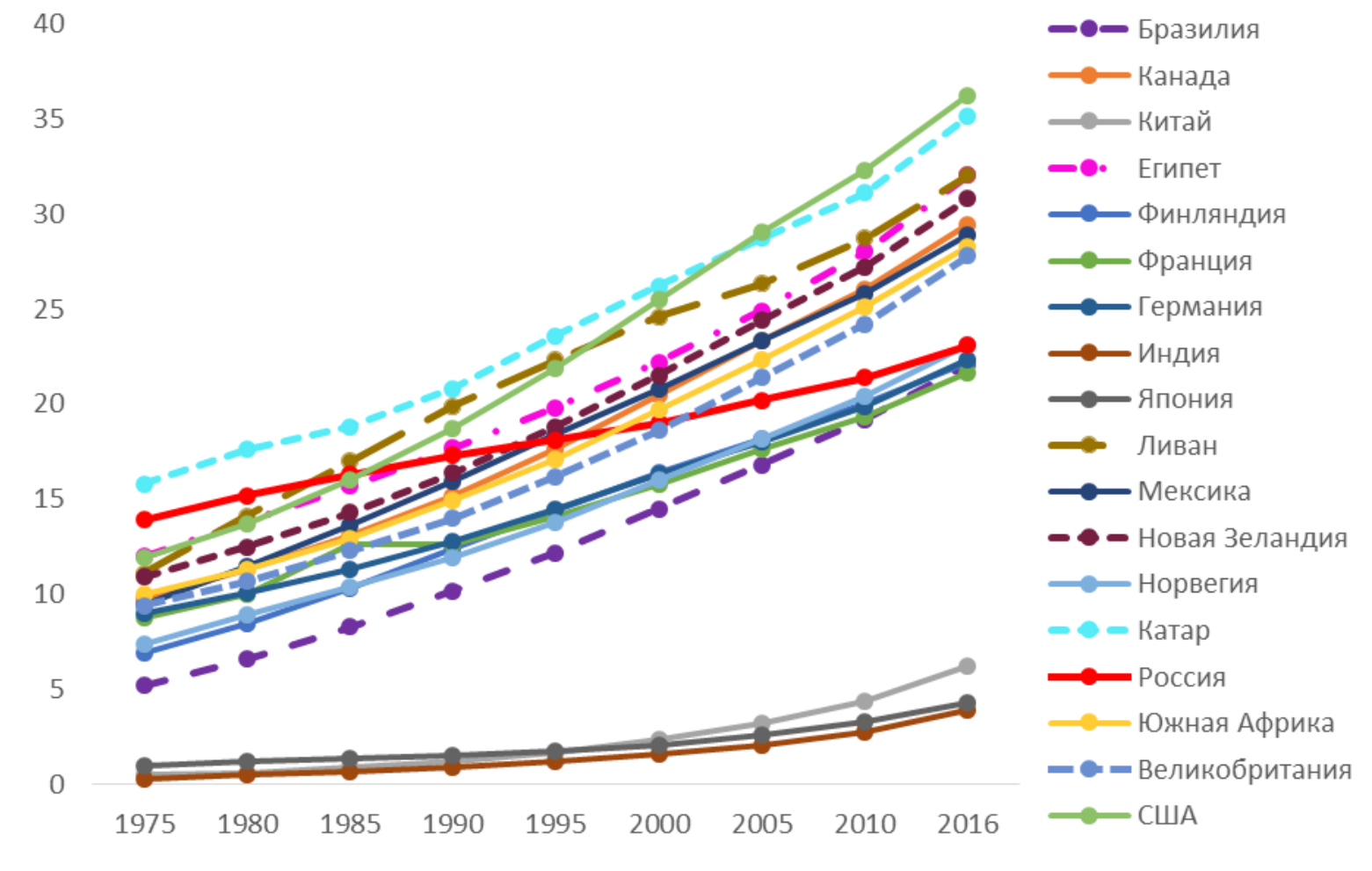

\section{Рисунок 1. Динамика доли взрослого населения (18+), страдающего ожирением,} 1975-2016, \%

Источник: Построено на основе данных WHO Global Health Observatory. Data Repository. URL: https://www.who.int/gho/database/en/ (дата обращения 19.08.2018).

Вполне естественно, что возникновение и осознание такой масштабной проблемы, имеющей серьезные социально-экономические последствия, вызвало интерес ученых, изучающих ожирение с самых разных позиций. Ожирение, как и в целом проблематика здорового образа жизни, - междисциплинарная тема и исследуется сегодня не только эпидемиологами, демографами, антропологами, но и психологами, социологами, экономистами. В российской социально-экономической литературе исследования по ожирению пока крайне немногочисленны. Так, в работе [Рощина, Гремченко 2016] показана на дескриптивном уровне связь между избыточным весом и показателями здоровья, дохода и образования россиян на данных за 1994-2014 гг.; в работе [Колосницына, Бердникова 2009] на основе пространственной выборки за 2006 г. обнаружена немонотонная зависимость индекса массы тела от возраста, а также неодинаковая связь избыточного веса и образования для мужчин и для женщин. Цель данной статьи представить обзор основных социально-экономических факторов и последствий

\footnotetext{
${ }^{2}$ URL: http://www.who.int/features/factfiles/obesity/en/ (дата обращения: 19.08.2018).
} 
избыточного веса и провести детальный анализ сложившейся в России ситуации с помощью данных Российского мониторинга экономического положения и здоровья населения НИУ ВШЭ.

\section{ПОНЯТИЯ ИЗБЫТОЧНОГО ВЕСА И ОЖИРЕНИЯ}

При анализе проблемы избыточного веса населения ВО3 использует стандартный показатель, называемый индексом массы тела (ИМТ; Body Mass Index - BMI ${ }^{3}$. Он представляет собой частное от деления веса человека в килограммах на квадрат его роста в метрах. Индекс используется для отнесения взрослого человека к той или иной категории:

ИМТ < 18,5 - недостаточный вес;

$18,5 \leq$ ИМТ $<25$ - нормальный вес;

$25 \leq$ ИМТ < 30 - избыточный вес (предожирение);

$30 \leq$ ИМТ - избыточный вес (ожирение).

Среди страдающих ожирением иногда выделяют подгруппы с ИМТ от 30 до 35, от 35 до 40, от 40 до 45 и выше 45, с тем чтобы анализировать отдельно так называемые морбидные формы ожирения. Для оценки избыточного веса и ожирения у детей используют специальные статистические показатели, которые учитывают как возраст ребенка с точностью до месяца, так и его рост. Однако в рамках данной работы мы будем анализировать только проблематику «взрослого» ожирения.

Итак, к группе взрослого населения с избыточным весом относят и тех, кто попадает в категорию «предожирение», и тех, кто страдает ожирением, т.е. всех, чей ИМТ больше или равен 25. Однако в статистическом анализе и в научных публикациях часто используют по отдельности ту или иную группу: тех, кто имеет избыточный вес, но еще не достиг стадии ожирения (предожирение), или наоборот, только тех, кто страдает ожирением.

Важно также отметить, что сам по себе показатель ИМТ не идеален и подвергается критике, поскольку не в состоянии учесть таких индивидуальных особенностей человека, как костная или мышечная масса. В медицинских оценках избыточного веса используются дополнительные измерения, позволяющие получить более точные результаты. Например, производится измерение окружности талии, используется соотношение окружности талии и роста. Показатель ИМТ часто критикуется и на том основании, что он завышает показатели избыточного веса для высоких людей и занижает - для низких. Поэтому, в частности, для людей монголоидной расы используют более строгие критерии: так, в Гонконге, Японии и Сингапуре установлен критерий ожирения, начиная со значения ИМТ, равного 25.

Однако ИМТ универсален и удобен при проведении массовых опросов и потому признан исследователями и широко используется в специальной литературе.

\footnotetext{
${ }^{3}$ URL: http://www.who.int/en/news-room/fact-sheets/detail/obesity-and-overweight (дата обращения: 19.08.2018). 


\section{СОЦИАЛЬНО-ЭКОНОМИЧЕСКИЕ ФАКТОРЫ И ПОСЛЕДСТВИЯ ИЗБЫТОЧНОГО ВЕСА: ОБЗОР ИССЛЕДОВАНИЙ}

Экономические исследования проблематики избыточного веса появились относительно недавно: в 1990-е годы были опубликованы первые работы в США, где впервые ученые столкнулись с таким массовым явлением. Однако затем, с распространением самого феномена ожирения сначала в развитых, а затем и в развивающихся странах, начали появляться работы с использованием собственных данных и все более сложных инструментальных методов. Международные организации, прежде всего ВОЗ и ОЭСР, занимаются сбором информации и межстрановыми сопоставлениями данных и результатов анализа, полученных в отдельных странах [Sassi et al. 2009].

\section{Причины и факторы ожсирения}

Разумеется, не все причины ожирения можно отнести к экономическим или - шире социальным. Люди, имеющие наследственную предрасположенность к ожирению, при прочих равных условиях с большей вероятностью страдают от избыточного веса [Stunkard et al. 1990]. Недавний обзор исследований, проведенный канадскими учеными, показал, что существуют значимые этнические различия в распространенности избыточного веса [Stryjecki, Alyass, Meyre 2018]. Однако наследственная предрасположенность никак не может объяснить быстрых темпов роста ожирения, наблюдаемых в течение последних десятилетий во всем мире, в том числе и в рамках отдельных этнических групп. Генетика определяет всего 1\% из всех случаев ожирения [Antonanzas, Rodriguez 2010: 351]. Точно так же нельзя объяснить растущие показатели ИМТ детерминантами пола и возраста. Действительно, в большинстве стран к ожирению более склонны женщины и люди старших возрастов [Sassi et al. 2009]: вес, как правило, растет с возрастом и начинает снижаться лишь в самом конце жизни, но так было всегда. Сегодня же мы наблюдаем все большую распространенность избыточного веса среди детей и подростков, которые переходят во взрослое состояние уже отягощенные этими проблемами [WHO 2017]. Сравнительные исследования, проведенные в странах ОЭСР, подтверждают: основные детерминанты исследуемого явления лежат в сфере жизнедеятельности человека [Sassi et al. 2009].

В большинстве случаев, как утверждают физиологи, ожирение возникает как результат дисбаланса потребляемой и расходуемой человеком энергии. Соответственно, экономисты ищут причины растущего избыточного веса как на стороне потребления пищи, так и на стороне физической активности современных людей в быту и на рабочем месте. Почему с некоторых пор человек стал есть больше, чем ему на самом деле требуется? Некоторые теории объясняют это сохранившейся в сознании человечества привычкой есть много тогда, когда пища в достатке, поскольку ее предложение ограничено и не гарантировано [Rosin 2008]. Другие авторы предлагают применять к пище известную теорию рациональной аддикции, первоначально объяснявшую потребление алкоголя, табака и наркотиков [Becker, Murphy 1988]. К аддиктивным благам относят сахар, соль, жиры. Причем привычку к этим вредным для организма продуктам (как и в случае с табаком и алкоголем) поддерживают производители и продавцы с помощью активного маркетинга, так что спрос во многом формируется предложением [Courtemanche, Carden 2011]. Покупатель, особенно юный, часто не осведомлен о вредных свойствах продуктов, 
что в экономике называют информационной асимметрией и относят к провалам рынка. Другой возможный провал рынка - специфика межвременных предпочтений потребителя в отношении пищи: при высокой индивидуальной ставке дисконтирования он не готов отказаться от удовольствия сегодня ради отложенных выгод в будущем, даже если имеет полную информацию [Komlos, Smith, Bogin 2004]. Важным фактором растущего спроса на пищу становятся и технологические изменения: приобретает все большую популярность готовая или полуготовая еда, на приготовление которой не надо тратить время. Современные технологии и развитие мировой торговли делают продукты питания относительно дешевыми, что, при прочих равных условиях, увеличивает потребление и избыточный вес [Grossman, Tekin, Wada 2014; Lu, Goldman 2010].

Другая сторона проблемы - снижение уровня физической активности современного человека. Это, с одной стороны, следствие изменений на рынке труда: занятость все более смещается в сферу услуг, большинство работ становятся офисными или даже домашними (дистанционными), а значит малоподвижными [Philipson, Posner 2003]. Кроме того, многие работники сталкиваются с высоким уровнем стресса, что также вносит вклад в растущие показатели ожирения [Geiker at al. 2018; Lallukka et al. 2008]. Трудоголики, которые проводят на работе все больше времени и все меньше спят, имеют больше шансов приобрести лишний вес [Courtemanche 2009]. С другой стороны, современные технологии упрощают быт и облегчают домашний труд. Вносит свой вклад и урбанизация: развитый транспорт и собственные автомобили позволяют городским жителям не ходить пешком [Eid et al. 2008]. Даже проведение досуга становится более пассивным, особенно это очевидно у детей: подвижные игры заменяются компьютерными. Чтобы поддерживать необходимый организму уровень физической активности, человек должен предпринимать специальные усилия: заниматься спортом в той или иной форме, причем регулярно, что требует времени и денег, а главное - воли.

Таким образом, результат - наличие ожирения или его отсутствие - во многом зависит от поведения самого человека, а также от условий, в которых он живет, учится, работает. Социально-экономические факторы, ассоциируемые с избыточным весом, многочисленны и разнообразны.

Многие работы указывают на образование как одну из детерминант веса. Так, например, в отчете ОЭСР показано, что в Англии 28\% женщин с образованием 8 классов страдают ожирением, тогда как среди проучившихся 15 лет и более эта доля всего $12 \%$. Авторы объясняют эту связь так: образованные люди лучше информированы о последствиях своего образа жизни, а потому более склонны к здоровому питанию, больше занимаются спортом и др. С другой стороны, дети, страдающие ожирением, чаще рано бросают учебу, их шансы получить хорошее образование меньше [Sassi 2010]. Таким образом, налицо порочный круг: как образование может определять показатели веса, так и наоборот. Сравнивая данные и исследования, проведенные в развитых странах ОЭСР, специалисты констатируют, что однозначная связь образования и веса прослеживается только для женщин, тогда как в отношении мужчин результаты неочевидны [Sassi et al. 2009]. Вместе с тем исследование, проведенное в Корее, указывает на положительную зависимость интенсивности занятий спортом от образования именно среди мужчин [Park, Kang, 2008]. 
Неоднозначная связь наблюдается между ожирением и доходами. На популяционном уровне зависимость меняет свой знак по мере достижения определенного уровня среднедушевых доходов: сначала рост благосостояния и среднего веса идут рука об руку, затем, после достижения определенного порога дохода, вес начинает снижаться [Sassi et al. 2009]. Многочисленные работы Рума, выполненные на данных США [Ruhm 2005], и аналогичные расчеты, выполненные в Исландии [Ásgeirsdóttir et al. 2015], показывают, что в развитых странах экономический спад, сопровождаемый падением доходов, приводит к снижению показателей веса.

Влияют на ожирение и социальные связи (семья, друзья, коллеги) [Christakis, Fowler 2007], а также окружающая среда - то, где человек живет, есть ли у него возможности ходить пешком, заниматься спортом рядом с домом, покупать здоровые продукты [CohenCole, Fletcher 2008].

\section{Последствия избыточного веса}

Вполне очевидно, что последствия избыточного веса анализируются прежде всего в контексте заболеваемости. Пионерной работой в этой области стало исследование X. Валера, который еще в 1984 г. показал на данных обширной лонгитюдной выборки населения Норвегии немонотонную зависимость уровня заболеваемости от веса, контролируя при этом возраст индивидов. Согласно его результатам, статистически значимый рост заболеваемости наблюдался в группах населения со слишком низкими и слишком высокими значениями ИМТ (ниже 21 и выше 29). Выявленная зависимость получила в литературе название "кривой Валера" [Waaler 1984]. Сама по себе связь заболеваний с ожирением - предмет медицинских исследований, однако экономистов здесь интересуют, как всегда, расходы (или упущенная выгода, что одно и то же). Многочисленные работы доказывают теоретически [Gupta, Greve 2011] и подтверждают эмпирически более высокие медицинские расходы полных людей [Withrow, Alter 2011], при том что другие индивидуальные характеристики контролируются. Такие расходы могут быть как частными, так и государственными, в зависимости от сложившейся в стране системы медицинской помощи. Они могут проявляться в разных формах и отражаться различными показателями: частота посещения врача или вызовов «скорой помощи», частота и продолжительность госпитализаций, стоимость лекарств, процедур, операций и др. По данным Международной ассоциации изучения ожирения (IASO), доля расходов национальных систем здравоохранения на лечение ожирения оценивается в развитых странах от 2 до $8 \%$ общего бюджета здравоохранения ${ }^{4}$, что больше, чем затраты на лечение онкологических больных.

Кроме прямых затрат на лечение заболеваний, связанных с ожирением, возникают многочисленные косвенные расходы, которые несут сам работник, работодатель, страховая компания или государство. В экономической литературе есть множество работ, подтверждающих связь между избыточным весом и положением работника на рынке труда. В частности, речь идет об отрицательной корреляции избыточного веса и заработков [Sargent et al. 1994]. Избыточный вес также может отражаться на возможностях занятости:

\footnotetext{
${ }^{4}$ URL: https://web.archive.org/web/20040824052003/http://www.iotf.org/ (дата обращения: 19.08.2018).
} 
вероятности найма, возможности занять лучшее рабочее место и/или удержаться в случае сокращений и др. Механизмы подобных связей могут быть различными. С одной стороны, избыточный вес может быть показателем плохого здоровья, которое, в свою очередь, делает работника менее продуктивным, а потому и оплата его труда оказывается относительно низкой в сравнении со здоровым коллегой. С другой стороны, избыточный вес и, в особенности, ожирение может стать причиной для дискриминации, когда работодатель или коллеги, или клиенты компании не хотят видеть такого работника, и его возможности трудоустройства оказываются ограниченными [Morris 2007]. В любом случае результатом становится так называемый «штраф» со стороны рынка труда.

Есть основания считать, что влияние ожирения на трудовые доходы различается для мужчин и женщин, высокооплачиваемых и низкооплачиваемых работников, а кроме того, оно может быть неодинаковым на разных этапах жизненного цикла работника и различаться для разных поколений. Так, самые ранние исследования этого феномена уже обнаруживали, что негативное влияние избыточного веса на заработки наиболее очевидно прослеживается для женщин, тогда как для мужчин оно статистически незначимо [Register, Williams 1990; Averett, Korenman 1996]. В недавнем исследовании Браун и Роутон, используя лонгитюдные данные и более сложную технику регрессионного анализа, выявили, что штраф за избыточный вес у женщин существенно возрастает по мере перемещения в высокодоходные группы, где он в несколько раз выше, чем у низкооплачиваемых работниц. В отличие от многих других авторов, они показали, что и для мужчин в некоторых доходных группах вес и заработки значимо и отрицательно связаны. Кроме того, оказалось, что штраф за лишний вес растет от поколения к поколению [Brown, Routon 2018].

В связи с большей вероятностью и продолжительностью заболеваний, полные работники чаще и дольше отсутствуют на рабочем месте по болезни, чаще получают травмы, инвалидность. Это дополнительные расходы, которые оплачиваются полностью или частично и несут убытки не только самому работнику, но и компании, и государству.

Можно привести примеры оценок, сделанных в разные годы и для разных стран. Так, в конце 1990-х годов в Китае дополнительный ущерб от пропусков работы людьми, страдающими ожирением, оценивался в 44,8 млрд долл. в годовом выражении [Popkin et al. 2006]. В 2000 г. в США затраты, связанные с пропусками по болезни, в расчете на одного человека, страдающего ожирением, превышали аналогичные расходы на работника с нормальным весом на 300-1000 долл. в зависимости от пола и степени ожирения [Filkenstein, Fiebelkorn, Wang 2005]. В 1992 г. во Франции дополнительные издержки из-за пропусков рабочего времени людьми, страдающими ожирением, оценивались в 66 млн долл. [Levy et al. 1995]. В начале 2000-х годов в США вероятность травмы на производстве оказалась в 1,26 и 1,76 раз выше у работников с избыточным весом и ожирением соответственно по сравнению с работниками с нормальным весом [Arena et al. 2006]. В США в 1994-2003 гг. средняя продолжительность пребывания в отпуске по болезни для работников-мужчин с ожирением была 10,5 дней против 6,8 дней у их коллег с нормальным весом; для женщин эти показатели составили 21,8 и 7,7 дней соответственно [Tsai et al. 2005]. 


\section{ЭМПИРИЧЕСКИЙ АНАЛИЗ ФАКТОРОВ И ПОСЛЕДСТВИЙ ИЗБЫТОЧНОГО ВЕСА В РОССИИ}

\section{Данные}

Исследование избыточного веса в России проводилось на базе данных Российского мониторинга экономического положения и здоровья населения (РМЭЗ) НИУ ВШЭ 5 . Анкета содержит вопросы о росте и весе респондента, что позволяет рассчитать его ИМТ, а также дает обширную информацию об индивидуальных характеристиках, включая здоровье, образование, семейный статус, наличие детей, место жительства, а также о занятости и зарплате работающих респондентов. Лонгитюдный характер мониторинга позволяет использовать панельные данные, что дает возможность учитывать индивидуальные особенности отдельных респондентов при построении регрессионных моделей.

Для дескриптивного анализа использовались данные с 1995 по 2016 г. (репрезентативные выборки за соответствующие годы), которые позволяют видеть ситуацию в динамике; более подробно изучались результаты 21-й - 25-й волн 2012-2016 г., с тем, чтобы проследить связь избыточного веса с отдельными характеристиками респондентов. Для построения регрессионных моделей также использовалась объединенная выборка РМЭЗ за 5 лет с 2012 по 2016 г., состоящая из респондентов в возрасте от 19 до 100 лет с ИМТ от 18 до 50. В анализируемых выборках в качестве взрослых людей мы оставляли только тех, чей возраст старше 18 лет (19+), поскольку, согласно принятой методике ВОЗ, в 19 лет «детский» подход к определению избыточного веса уже совпадает со «взрослым» ${ }^{6}$. Таким образом, мы могли использовать показатель ИМТ для всех респондентов в этой выборке. Число наблюдений составило 54515 (31999 относительно женщин и 22516 относительно мужчин). Данные представляют собой несбалансированную панель, в которую вошли все респонденты, проходившие опрос хотя бы один раз за этот период. Полученная таким образом панель состоит из результатов опроса 17981 индивида. В оцениваемых далее регрессионных моделях размеры панельной выборки уменьшались, так как, в зависимости от целей анализа, рассматривались либо только работающие индивиды, либо лица трудоспособного возраста, и не все респонденты отвечали на необходимые вопросы.

\section{Дескриптивный анализ}

Чтобы проследить тенденции динамики избыточного веса, был рассчитан средний индекс массы тела в целом по выборке и отдельно для мужчин и женщин. На рисунке 2 показан очевидный рост этих показателей (сплошными линиями - средние значения ИМТ для мужчин, женщин и в среднем по выборке, пунктирными линиями - соответствующие 95\%ные доверительные интервалы). Уже в 1995 г. средний взрослый человек в России

\footnotetext{
5 Российский мониторинг экономического положения и здоровья населения НИУ-ВШЭ (RLMS-HSE), проводимый Национальным исследовательским университетом "Высшая школа экономики" и ООО «Демоскоп» при участии Центра народонаселения Университета Северной Каролины в Чапел Хилл и Института социологии Федерального научно-исследовательского социологического центра РАН. (Сайты обследования RLMS-HSE: http://www.cpc.unc.edu/projects/rlms и http:/www.hse.ru/rlms).

${ }^{6}$ URL: http://www.who.int/growthref/who2007_bmi_for_age/en/ (дата обращения: 19.08.2018).
} 
находился в категории предожирения: его ИМТ был выше 25. Средний мужчина достиг порога избыточного веса только в 2000 г., однако в течение последних десяти лет по этому показателю он постепенно догоняет среднюю женщину: за 20 лет с 1995 по 2016 г. разрыв в ИМТ между ними сократился на 0,4 единицы. Эти изменения происходят, как нетрудно понять, за счет роста доли мужского населения, имеющего избыточный вес.

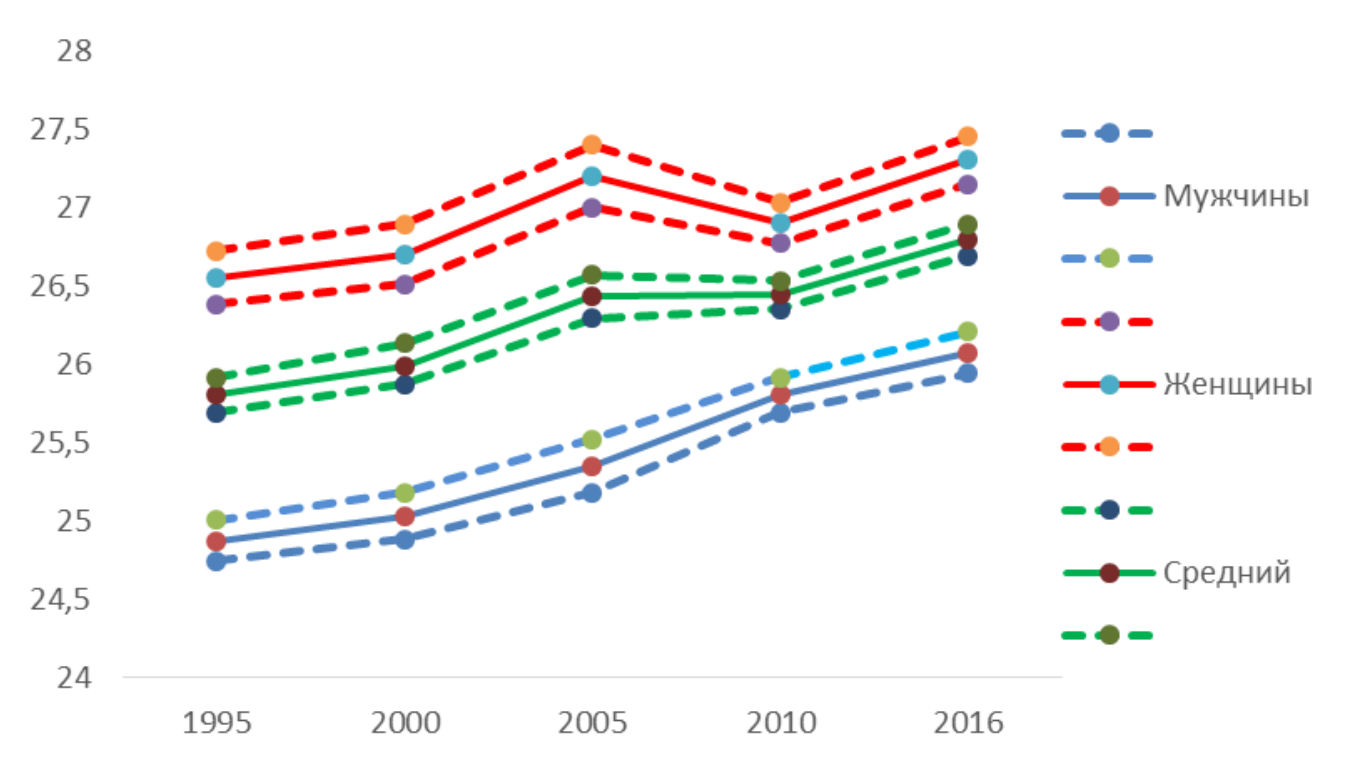

Рисунок 2. Динамика среднего ИМТ взрослого населения, 1995-2016

Источник: Расчеты авторов на основе данных РМЭЗ НИУ ВШЭ.

При этом, если доля тех, кто относится к категории предожирения (ИМТ больше 25, но меньше 30), достаточно стабильна - 34,7\% в 1995 г. и 35,4\% в 2016 г., то доля страдающих ожирением за этот же период выросла радикально: с 19,2 до 26,7\%, или в 1,4 раза (рисунок 3). В результате избыточный вес в 2016 г. имело более половины взрослого населения страны старше 18 лет - 62,2\%.

На рисунке 4 показано, как изменялась ситуация в гендерном разрезе. И в 1995 г., и в 2016 г. (как и во все остальные годы внутри этого периода) предожирение было более характерно для мужчин, а ожирение - для женщин. В этом смысле Россия не исключение, в большинстве развитых стран наблюдается именно такая картина [Sassi et al. 2009]. Но важно отметить, что ожирение растет более быстрыми темпами именно среди мужчин, и в этой же гендерной группе увеличивается предожирение, тогда как среди женщин предожирение стабильно, а ожирение увеличивается не так стремительно. Если подобные тенденции сохранятся, в перспективе возможно сглаживание гендерных различий. 


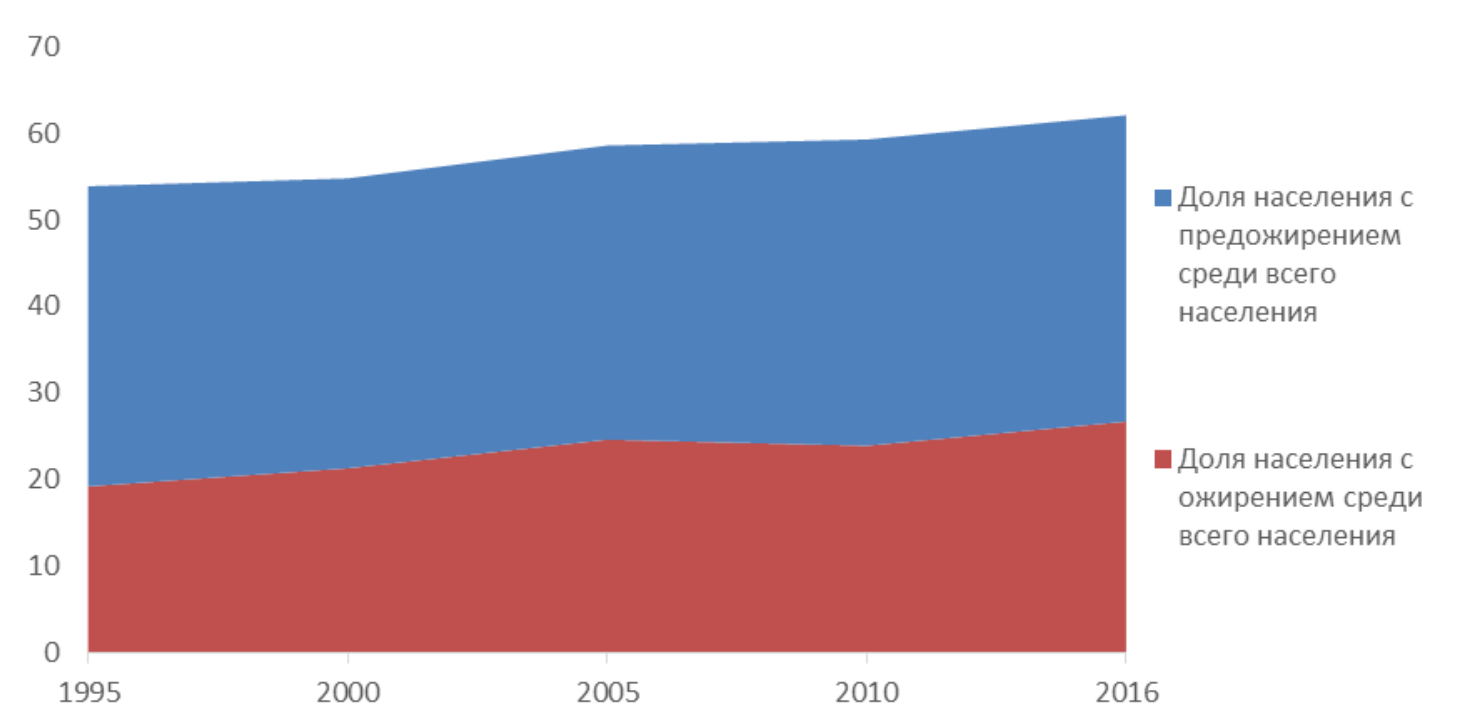

Рисунок 3. Динамика доли взрослого населения, страдающего предожирением и ожирением, 1995-2016, \%

Источник: Расчеты авторов на основе данных РМЭЗ НИУ ВШЭ.

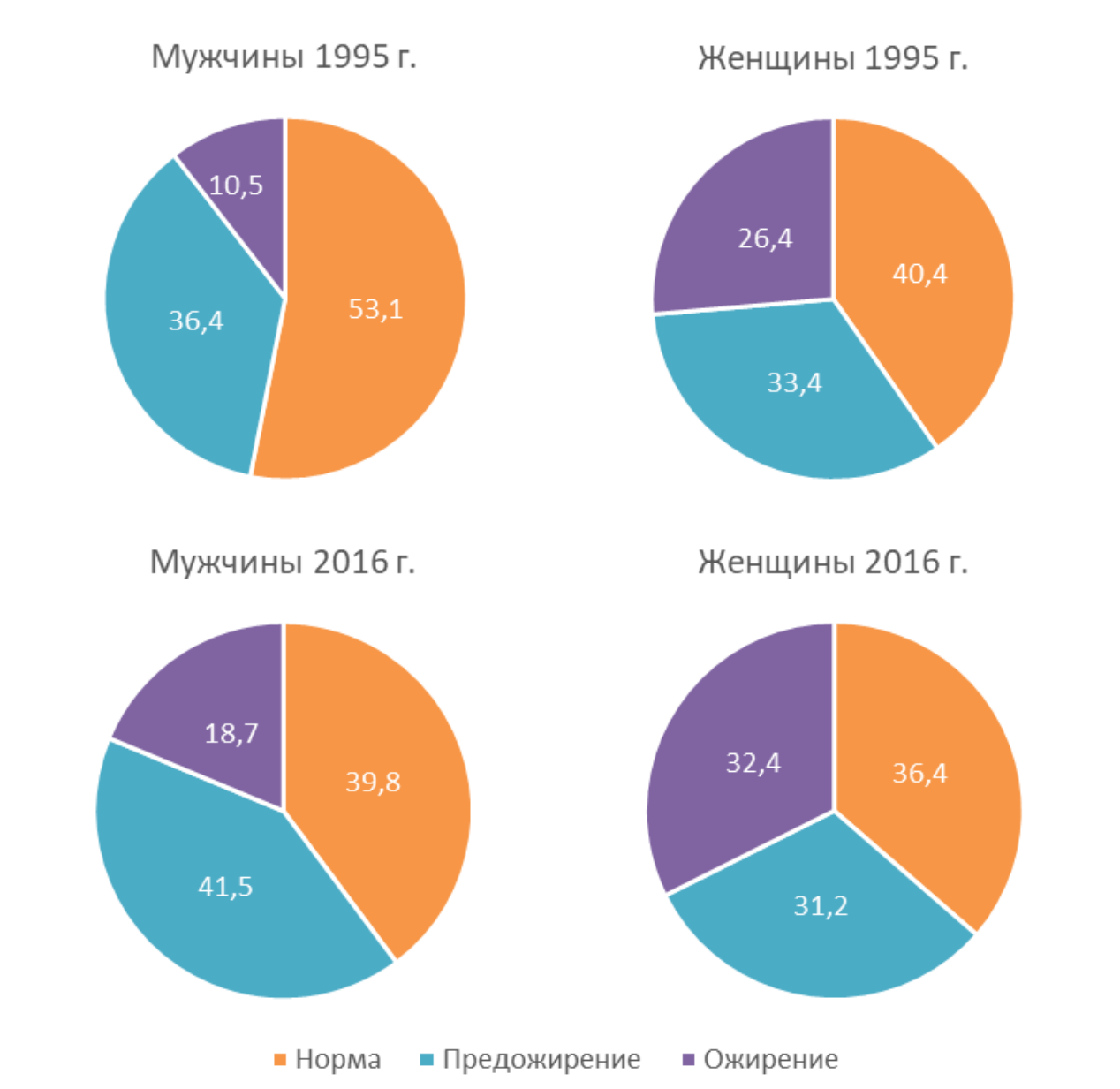

Рисунок 4. Распределение мужчин и женщин по группам в соответствии с весом, 1995 и 2016 гг., \% (в 1995 г. N=6626; в 2016 г. N=9506)

Источник: Расчеты авторов на основе данных РМЭЗ НИУ ВШЭ. 
На данных 2012-2016 гг. можно проследить вполне ожидаемые различия распространенности избыточного веса с возрастом (рисунок 5). При этом и здесь отчетливо видна гендерная специфика. Во всех возрастных группах избыточный вес в целом более характерен для мужчин, чем для женщин. Вплоть до 40 лет большинству женщин удается поддерживать нормальный вес, тогда как у мужчин уже в группе 31-40 лет таковых остается всего 39\%, а ожирением страдают 43\%. Но в группе 41-50 лет у женщин резко возрастает ожирение, и этот процесс продолжается после 50 лет, так что в самой старшей возрастной группе мы наблюдаем обратную картину: среди женщин нормальный вес сохраняется лишь у $18 \%$, а среди мужчин - у $30 \%$.

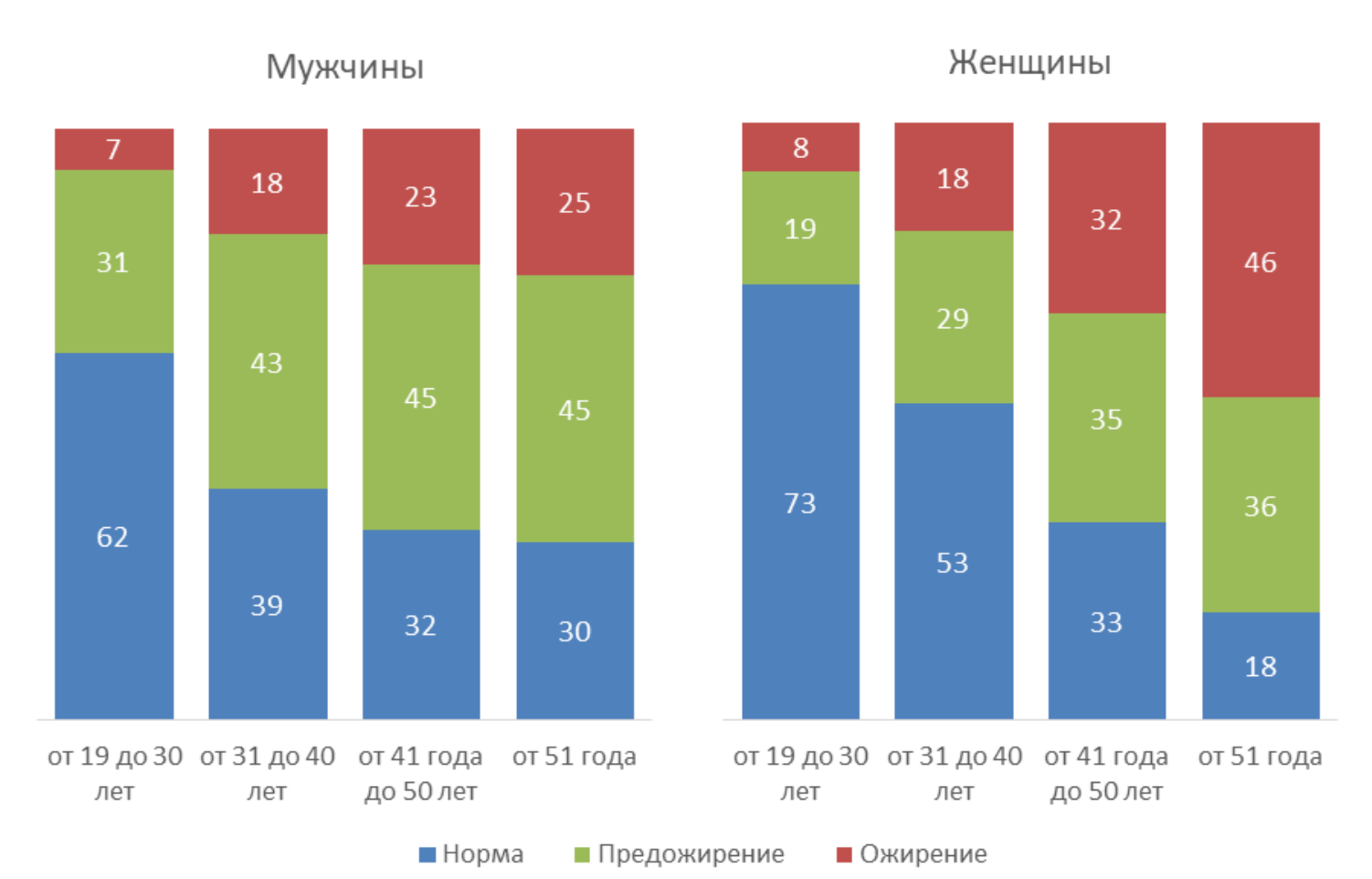

\section{Рисунок 5. Распределение респондентов по группам ИМТ внутри возрастных групп, 2012-2016, \%, $\left(\mathrm{N}_{\text {жен }}=31517, \mathrm{~N}_{\text {муж }}=22401\right)$}

Источник: Расчеты авторов на основе данных РМЭЗ НИУ ВШЭ.

Связь веса и образования также оказалась неодинаковой для мужчин и для женщин (рисунок 6). Все респонденты были разбиты на 4 группы по уровню образования: 1 - ниже среднего; 2 - законченное среднее, в том числе начальное профессиональное на базе среднего; 3 - среднее специальное; 4 - высшее и послевузовское. У мужчин образование и вес увеличиваются параллельно: по мере перемещения из группы с незаконченным средним образованием в каждую следующую группу вплоть до высшего, показатели ожирения и предожирения монотонно растут и соответственно снижается доля тех, кто сохраняет нормальный вес. Среди мужчин с высшим образованием всего $35 \%$ имеют нормальный вес, тогда как в группе не закончивших школу их 43\%. 


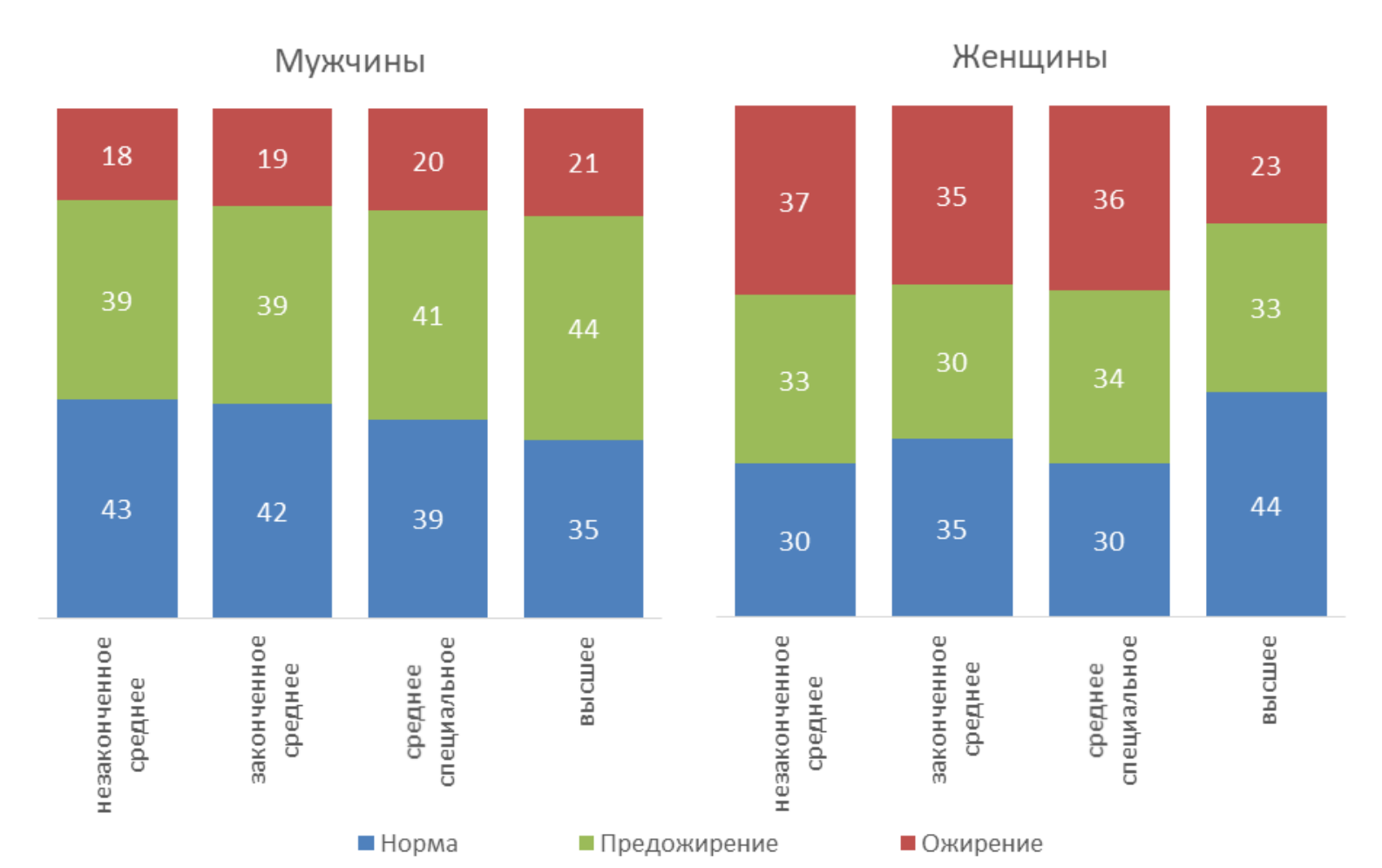

\section{Рисунок 6. Распределение респондентов по группам ИМТ внутри групп с разным уровнем образования, 2012-2016, \% $\left(\mathrm{N}_{\text {жен }}=\mathbf{2 5 8 5 5}, \mathrm{N}_{\text {муж }}=18400\right)$}

Источник: Расчеты авторов на основе данных РМЭЗ НИУ ВШЭ.

Иная ситуация среди женщин. Если в первых трех образовательных группах наблюдаются примерно одинаковые доли тех, кто страдает как ожирением, так и предожирением, то переход в группу с высшим образованием резко сокращает показатели ожирения (в 1,5 раза). В итоге среди женщин с высшим образованием доля сохранивших нормальный вес намного выше, чем в любой другой группе - 44\%. И наоборот, среди самых образованных женщин наиболее низкий уровень ожирения: 23\% против $35-37 \%$ в других группах. Любопытно, что этот результат полностью совпадает с полученным ранее на данных РМЭЗ за 2006 г.: значения показателей ожирения и предожирения возросли за прошедшие годы во всех образовательных и гендерных группах, но пропорции остались прежними - значимым фактором снижения веса выступает только высшее образование и только для женщин [Колосницына, Бердникова 2009: 85].

Ниже, в таблицах 1-3, приводятся основные средние характеристики респондентов в зависимости от их принадлежности к той или иной группе по весу. Они дают возможность проследить некоторые связи между избыточным весом и отдельными социальноэкономическими факторами.

Для построения таблицы 1 использовалась полная выборка за 2012-2016 гг., в которую вошли респонденты в возрасте 19 лет и старше (до 100 лет), ответившие на вопросы о возрасте, весе, росте и половой принадлежности. Число наблюдений в выборке составило 53918. Таблица показывает, какая доля респондентов в каждой весовой/гендерной группе обладает теми или иными характеристиками (например, отличаются хорошим здоровьем или состоят в браке). 
Таблица 1. Основные социально-демографические характеристики респондентов в зависимости от веса, 2012-2016

\begin{tabular}{|c|c|c|c|c|c|c|}
\hline \multirow[b]{2}{*}{ 2012-2016 гг. } & \multicolumn{3}{|c|}{ Мужчины } & \multicolumn{3}{|c|}{ Женщины } \\
\hline & норма & $\begin{array}{c}\text { пред- } \\
\text { ожирение }\end{array}$ & ожирение & норма & $\begin{array}{c}\text { пред- } \\
\text { ожирение }\end{array}$ & ожирение \\
\hline Средний возраст, лет & 41 & 47 & 50 & 41 & 54 & 58 \\
\hline Самооценка здоровья, \%: & & & & & & \\
\hline очень хорошее & 3,1 & 2,4 & 1,5 & 1,8 & 0,8 & 0,3 \\
\hline хорошее & 43,5 & 39,8 & 29,1 & 41,9 & 25,0 & 13,4 \\
\hline среднее & 44,1 & 49,0 & 55,0 & 46,9 & 57,6 & 62,7 \\
\hline плохое & 8,0 & 7,9 & 13,1 & 8,1 & 15,0 & 21,2 \\
\hline очень плохое & 1,4 & 1,0 & 1,4 & 1,3 & 1,7 & 2,5 \\
\hline $\begin{array}{l}\text { Состоят в браке } \\
\text { (зарегистрированном или } \\
\text { незарегистрированном), \% }\end{array}$ & 62,9 & 80,2 & 86,3 & 54,3 & 56,4 & 55,1 \\
\hline $\begin{array}{l}\text { Живут в городе или областном } \\
\text { центре, \% }\end{array}$ & 67,5 & 65,9 & 65,5 & 72,6 & 68,9 & 65,9 \\
\hline Статус занятости, \%: & & & & & & \\
\hline $\begin{array}{l}\text { учатся на дневной форме } \\
\text { обучения }\end{array}$ & 4,6 & 1,4 & 0,4 & 5,4 & 0,6 & 0,1 \\
\hline работают & 61,3 & 65,1 & 65,5 & 60,6 & 50,0 & 42,9 \\
\hline пенсионеры & 16,4 & 20,7 & 24,0 & 17,0 & 38,4 & 48,9 \\
\hline Имеют высшее образование, \% & 20,7 & 25,2 & 25,3 & 35,3 & 28,9 & 19,7 \\
\hline Число наблюдений, чел. & 8920 & 9189 & 4292 & 11226 & 10112 & 10179 \\
\hline
\end{tabular}

Источник: Расчеты авторов.

Примечание: Все различия в показателях между выделенными группами статистически значимы на 5\%-ном уровне.

Из таблицы видно, что самооценка здоровья респондентов ухудшается по мере перехода из групп с нормальным весом к группам с ожирением, причем среди женщин эта тенденция выражена сильнее. Очевидно, здесь играет свою роль и возраст, который растет параллельно весу. Среди мужчин, страдающих ожирением, существенно выше доля состоящих в браке, тогда как среди женщин такой связи на уровне средних показателей не видно. Для обеих гендерных групп среди тех, чей вес в норме, выше доля горожан. Это также может объясняться возрастными различиями и тем, что среди людей с нормальным весом большой процент учащихся, в том числе студентов, проживающих в городах. Среди женщин доля занятых на рынке труда падает с увеличением веса, а среди мужчин, напротив, растет. Доля имеющих высшее образование больше всего среди самых полных мужчин, тогда как у женщин все ровно наоборот: среди страдающих ожирением процент закончивших вуз самый низкий.

В таблице 2 показаны характеристики работающих респондентов также в зависимости от их принадлежности к «весовым» группам. Для построения этой таблицы в выборке 2012-2016 гг. были оставлены только респонденты, работавшие на момент опроса или находившиеся в отпуске, число наблюдений составило 29266. Доля респондентов, пропускавших работу по болезни, не демонстрирует какой-либо связи с весом ни у мужчин, ни у женщин, видимо из-за специфики профессиональной деятельности. Число дней, проведенных на больничном теми, кто все-таки пропускал работу, немного растет при переходе в группы с избыточным весом. Мы также видим некоторое снижение средней заработной платы с ростом веса у женщин, тогда как у мужчин картина обратная: заработки 
выше всего в группе страдающих ожирением (все зарплаты приведены к ценам 2016 г.). Однако статистически значимыми на 5\%-ном уровне оказались только различия в заработной плате между всеми выделенными группами.

Таблица 2. Основные характеристики работающих респондентов в зависимости от веса, 2012-2016

\begin{tabular}{|c|c|c|c|c|c|c|}
\hline & \multicolumn{3}{|c|}{ Мужчины } & \multicolumn{3}{|c|}{ Женщины } \\
\hline & норма & $\begin{array}{c}\begin{array}{c}\text { пред- } \\
\text { ожирение }\end{array} \\
\end{array}$ & ожирение & норма & $\begin{array}{c}\begin{array}{c}\text { пред- } \\
\text { ожирение }\end{array} \\
\end{array}$ & ожирение \\
\hline $\begin{array}{l}\text { Средняя заработная плата } \\
\text { в месяц, руб*. }\end{array}$ & 28507 & 30831 & 33059 & 23423 & 22094 & 19451 \\
\hline $\begin{array}{l}\text { Пропускали работу по } \\
\text { болезни в течение года, \% }\end{array}$ & 17,2 & 16,0 & 15,8 & 20,1 & 19,6 & 21,6 \\
\hline $\begin{array}{l}\text { Пропущено по болезни в } \\
\text { среднем в течение года, } \\
\text { дней** }\end{array}$ & 15 & 17 & 19 & 15 & 18 & 20 \\
\hline Число наблюдений, чел. & 5436 & 5961 & 2792 & 6187 & 4720 & 4170 \\
\hline
\end{tabular}

Источник: Расчеты авторов.

Примечания: * - в ценах 2016 г.; ** - среди пропускавщих работу по болезни.

Наконец, в таблице 3 приведена описательная статистика, касающаяся здоровья респондентов в зависимости от веса. Как и для построения таблицы 1, в данном случае использовали полную выборку за 2012-2016 гг. (53918 наблюдений). Вероятность госпитализации за последние три месяца, предшествовавшие опросу, несколько возрастает в группах населения с избыточным весом по сравнению с теми, чей вес в норме. Однако средняя продолжительность госпитализаций, как и среднее число визитов к врачу за год, не демонстрируют явной связи с весом респондентов.

На дескриптивном уровне прослеживается рост доли мужчин и женщин, покупавших лекарства за последний месяц, по мере увеличения веса. Соответственно увеличивается и средняя денежная сумма, потраченная за месяц на лекарственные средства (для сопоставимости все расходы пересчитаны в цены 2016 г.). И это неудивительно, поскольку избыточный вес, как уже отмечалось, ассоциирован с более частыми заболеваниями, в том числе хроническими. В таблице 3 показан рост доли респондентов, имеющих любые хронические заболевания, по мере перемещения в группы страдающих избыточным весом. Особенно отчетливо эти различия прослеживаются для заболеваний сердца, гипертонии, диабета. Логическим продолжением увеличения заболеваемости становится растущая инвалидизация: среди мужчин с ожирением доля инвалидов в 1,5 раза выше, чем среди мужчин с нормальным весом; у женщин инвалидность в группе с ожирением встречается почти в 3 раза чаще, чем в группе с нормальным весом. Разумеется, наблюдаемые на уровне средних величин различия в показателях здоровья между «весовыми» группами могут быть частично объяснены разницей среднего возраста респондентов и других характеристик, поэтому для выявления фактора веса требуются более точные оценки. 
Таблица 3. Характеристики здоровья респондентов в зависимости от веса, 2012-2016

\begin{tabular}{|c|c|c|c|c|c|c|}
\hline & \multicolumn{3}{|c|}{ Мужчины } & \multicolumn{3}{|c|}{ Женщины } \\
\hline & норма & $\begin{array}{c}\text { пред- } \\
\text { ожирение }\end{array}$ & ожирение & норма & $\begin{array}{c}\text { пред- } \\
\text { ожирение }\end{array}$ & ожирение \\
\hline $\begin{array}{l}\text { Лежали в больнице за } \\
\text { последние } 3 \text { месяца, \% }\end{array}$ & 4,0 & 3,9 & 4,6 & 4,5 & 5,6 & 6,7 \\
\hline $\begin{array}{l}\text { Среднее число дней в } \\
\text { больнице за последние } 3 \\
\text { месяца }\end{array}$ & 17,8 & 16,2 & 15,1 & 13,6 & 13,6 & 13,7 \\
\hline $\begin{array}{l}\text { Средняя частота посещения } \\
\text { врача за год, раз }\end{array}$ & 4,1 & 4,0 & 3,9 & 3,7 & 3,6 & 3,4 \\
\hline $\begin{array}{l}\text { Покупали лекарства для } \\
\text { лечения дома за последний } \\
\text { месяц, \% }\end{array}$ & 31,1 & 35,4 & 41,4 & 45,6 & 57,3 & 67,5 \\
\hline $\begin{array}{l}\text { Расходы на лекарства за } \\
\text { последний месяц, руб.* }\end{array}$ & 1525 & 1571 & 1724 & 1533 & 1693 & 1801 \\
\hline $\begin{array}{l}\text { Есть хронические } \\
\text { заболевания, \%: }\end{array}$ & & & & & & \\
\hline сердца & 9,4 & 12,1 & 19,7 & 10,3 & 20,8 & 30,0 \\
\hline легких, бронхов & 7,2 & 5,8 & 6,8 & 5,1 & 7,5 & 10,2 \\
\hline печени & 5,2 & 5,4 & 6,3 & 5,7 & 11,0 & 16,4 \\
\hline почек & 4,3 & 5,0 & 7,2 & 8,0 & 11,1 & 13,9 \\
\hline $\begin{array}{l}\text { желудочно-кишечного } \\
\text { тракта }\end{array}$ & 15,3 & 15,2 & 15,9 & 18,2 & 23,1 & 25,1 \\
\hline позвоночника & 14,8 & 17,0 & 19,8 & 14,4 & 22,7 & 27,5 \\
\hline $\begin{array}{l}\text { эндокринной системы, } \\
\text { сахарный диабет или } \\
\text { повышенный сахар в } \\
\text { крови }\end{array}$ & 1,4 & 3,1 & 8,7 & 5,6 & 12,1 & 21,3 \\
\hline $\begin{array}{l}\text { гипертоническая болезнь, } \\
\text { повышенное артериальное } \\
\text { давление }\end{array}$ & 11,7 & 20,8 & 35,7 & 15,0 & 36,6 & 57,3 \\
\hline заболевания суставов & 12,8 & 15,8 & 21,7 & 14,8 & 29,7 & 42,2 \\
\hline $\begin{array}{l}\text { неврологические } \\
\text { заболевания }\end{array}$ & 5,3 & 5,5 & 6,7 & 6,2 & 9,0 & 11,1 \\
\hline $\begin{array}{l}\text { варикозное расширение } \\
\text { вен }\end{array}$ & 3,4 & 4,4 & 6,6 & 7,6 & 14,9 & 21,8 \\
\hline $\begin{array}{l}\text { онкологические } \\
\text { заболевания }\end{array}$ & 1,0 & 1,1 & 1,3 & 1,3 & 2,6 & 3,4 \\
\hline $\begin{array}{l}\text { Среднее число хронических } \\
\text { заболеваний }\end{array}$ & 2,8 & 3,0 & 3,4 & 2,9 & 3,7 & 4,3 \\
\hline Назначена инвалидность, \% & 8,7 & 10,0 & 12,1 & 6,1 & 11,5 & 17,1 \\
\hline Число наблюдений, чел. & 8920 & 9189 & 4292 & 11226 & 10112 & 10179 \\
\hline
\end{tabular}

Источник: Расчеты авторов.

Примечания: * - В ценах 2016 г. Все различия в показателях между выделенными группами статистически значимы на 5\%-ном уровне.

Приведенные выше обзор научной литературы и дескриптивный анализ микроданных РМЭЗ НИУ ВШЭ позволили выдвинуть некоторые гипотезы эмпирического исследования избыточного веса населения России.

1. Избыточный вес населения в России, как и в других странах, связан с демографическими (пол, возраст) и социально-экономическими характеристиками (место жительства, семейное положение, образование, статус занятости, благосостояние). 
2. Избыточный вес связан со стилем питания, вредными и полезными привычками (курение, потребление алкоголя, занятия спортом).

3. Избыточный вес коррелирует с положением работника на рынке труда.

4. Наличие избыточного веса увеличивает при прочих равных условиях индивидуальные затраты на лечение.

5. Связь отдельных социально-экономических факторов с избыточным весом неодинакова для мужчин и для женщин.

\section{Моделирование и результаты}

Для проверки выдвинутых гипотез в работе используется регрессионный анализ панельных данных. К сожалению, проблема данных РМЭЗ, как и любых лонгитюдных обследований истощение выборки. Реальная панель оказывается слишком малой по объему, и при этом дополнительно возникают подозрения в ее смещенности ("выпадающие" год за годом респонденты, скорее всего, обладают характеристиками, отличными от генеральной совокупности). Поэтому мы используем панельную структуру данных при построении регрессионных моделей, но берем несбалансированную панель. Это, во-первых, увеличивает объем выборки, во-вторых, позволяет надеяться на меньшую смещенность, а в-третьих (за счет регрессионного анализа), позволяет контролировать одновременно многие важные характеристики респондентов. Эконометрический анализ позволяет оценивать степень связи изучаемой переменной с каждой из выбранных независимых переменных по отдельности, удерживая другие факторы постоянными.

Для проверки гипотез 1 и 2 использовалась Модель 1, оценивающая связь веса респондента с рядом демографических и социально-экономических факторов. Для проведения регрессионного анализа были сформированы следующие переменные.

Зависимая переменная:

- $\operatorname{lnbmi-~логарифм~ИМТ.~}$

Независимые переменные:

- male - пол, принимает значение 1 для мужчин, 0 для женщин;

- age/10 - возраст (число полных лет респондента, деленное на 10);

- $a g e^{2} / 100$ - возраст в квадрате (число полных лет респондента в квадрате, деленное на 100);

- $\quad s c h 1$ - максимальный завершенный уровень образования, принимает значение: 1 - для законченного среднего образования (в том числе начального профессионального образования - НПО - на базе среднего); 0 - во всех остальных случаях;

- $\quad s c h 2$ - максимальный завершенный уровень образования, принимает значение: 1 - для среднего специального образования; 0 - во всех остальных случаях;

- $s c h 3$ - максимальный завершенный уровень образования, принимает значение: 1 - для высшего и послевузовского образования; 0 - во всех остальных случаях;

- marriage - семейное положение, принимает значение: 1 - если респондент состоит в зарегистрированном или гражданском браке; 0 - в иных случаях; 
- $\quad$ city - тип населенного пункта, принимает значение: 1 - если респондент проживает в городе или областном центре; 0 - если респондент проживает в селе или поселке городского типа (ПГТ);

- capital - переменная столичного региона ${ }^{7}$, принимает значение: 1 - если респондент проживает в Ленинградской области, Санкт-Петербурге, Москве, Новой Москве или Московской области; 0 - во всех остальных случаях;

- child_- число несовершеннолетних детей, принимает значения от 0 до 9;

- $\quad$ smoke - принимает значение 1 , если респондент курил на момент опроса, 0 - иначе;

- $a l k$ - принимает значение 1 , если респондент употребляет хотя бы иногда алкогольные напитки, включая пиво, 0 - если нет;

- eat_h - частота питания вне дома, принимает значения: 1 - если респондент практически всегда питается вне дома; 2 - если все рабочие дни питается вне дома; 3 если 2-3 раза в неделю питается вне дома; 4 - если меньше двух раз; 5 - если питается только дома;

- eat_reg - удается ли питаться изо дня в день регулярно, не реже трех раз в день, принимает значения: 1 - если удается; 2 - если скорее да, чем нет; 3 - если скорее нет, чем да; 4 - если никогда не удается;

- $\quad$ sport - принимает значения: 0 - если респондент физкультурой не занимается; 1 - если выполняет легкие физкультурные упражнения для отдыха менее трех раз в неделю; 2 физкультурные упражнения средней или высокой тяжести менее трех раз в неделю; 3 физкультурные упражнения средней или высокой тяжести по крайней мере 3 раза в неделю по 15 минут и более; 4 - в случае ежедневных занятий физкультурой по меньшей мере 30 минут в день;

- employment - занятость, принимает значения: 1 - если респондент работает или находится в отпуске; 0 - если не работает;

- wealth - субъективная оценка материального благосостояния, принимает значения от 1 (низшая ступень) до 9 (высшая ступень);

- $y e a r-$ год проведения опроса.

Для проведения анализа в этом случае в панельную выборку вошли респонденты, которые смогли ответить на все необходимые вопросы. Таким образом, количество наблюдений за 2012-2016 гг. составило 41720, число респондентов - 13474. Проверка выбранных переменных на мультиколлинеарность не показала тесной связи, что позволило включить в модели все переменные одновременно. Оценивали модели логарифма ИМТ с фиксированными и случайными индивидуальными эффектами для всей выборки и отдельно для подвыборок мужчин и женщин. Все модели оказались статистически значимыми. Лучшие результаты, с точки зрения значимости коэффициентов и тесноты связи, продемонстрировали модели со случайными индивидуальными эффектами, что вполне обосновано с теоретической точки зрения, поскольку мы работали с данными по

\footnotetext{
${ }^{7}$ Предполагается, что проживание в столичном регионе значительно изменяет образ жизни респондентов за счет более продолжительной дороги на работу, интенсивного использования личных автомобилей или общественного транспорта, стрессов и др.
} 
большой совокупности индивидов за 5 лет. Эти модели и были выбраны для анализа. Результаты оценки представлены в таблице 4 (Модель 1).

\section{Таблица 4. Модель 1: зависимость ИМТ от демографических и социально- экономических характеристик}

\begin{tabular}{|c|c|c|c|}
\hline & Вся выборка & Мужчины & Женщины \\
\hline \multicolumn{4}{|l|}{ Переменные: } \\
\hline male & $\begin{array}{r}-0,019 * \\
(0,003)\end{array}$ & - & - \\
\hline age/10 & $\begin{array}{c}0,144^{*} \\
(0,004)\end{array}$ & $\begin{array}{r}0,1 * \\
(0,006)\end{array}$ & $\begin{array}{c}0,181^{*} \\
(0,005)\end{array}$ \\
\hline $\operatorname{age}^{2} / 100$ & $\begin{array}{r}-0,011 * \\
(0,0004)\end{array}$ & $\begin{array}{r}-0,008^{*} \\
(0,0006)\end{array}$ & $\begin{array}{r}-0,013^{*} \\
(0,0005)\end{array}$ \\
\hline \multicolumn{4}{|c|}{ Образование (база - незаконченное среднее): } \\
\hline $\operatorname{sch} 1$ & $\begin{array}{r}-0,002 \\
(0,002)\end{array}$ & $\begin{array}{r}0,001 \\
(0,003)\end{array}$ & $\begin{array}{r}0,005 \\
(0,003)\end{array}$ \\
\hline $\operatorname{sch} 2$ & $\begin{array}{r}0,003 \\
(0,003)\end{array}$ & $\begin{array}{r}0,008 * * \\
(0,003)\end{array}$ & $\begin{array}{r}-0,003 * * \\
(0,004)\end{array}$ \\
\hline $\operatorname{sch} 3$ & $\begin{array}{r}-0,013^{*} \\
(0,003)\end{array}$ & $\begin{array}{c}0,015^{*} \\
(0,004)\end{array}$ & $\begin{array}{r}-0,026^{*} \\
(0,004)\end{array}$ \\
\hline marriage & $\begin{array}{c}0,011 * \\
(0,002)\end{array}$ & $\begin{array}{c}0,018 * \\
(0,003)\end{array}$ & $\begin{array}{c}0,016 * \\
(0,002)\end{array}$ \\
\hline city & $\begin{array}{c}-0,018^{*} \\
(0,003)\end{array}$ & $\begin{array}{c}-0,012 * \\
(0,004)\end{array}$ & $\begin{array}{r}-0,025^{*} \\
(0,004)\end{array}$ \\
\hline capital & $\begin{array}{r}0,004 \\
(0,004)\end{array}$ & $\begin{array}{r}0,010 * * \\
(0,005)\end{array}$ & $\begin{array}{r}-0,003 * * \\
(0,005)\end{array}$ \\
\hline child & $\begin{array}{r}0,002 * * \\
(0,001)\end{array}$ & $\begin{array}{r}0,004 * * \\
(0,002)\end{array}$ & $\begin{array}{r}0,003 * * \\
(0,001)\end{array}$ \\
\hline smoke & $\begin{array}{r}-0,014 * \\
(0,002)\end{array}$ & $\begin{array}{c}-0,017 * \\
(0,002)\end{array}$ & $\begin{array}{c}-0,008 * \\
(0,003)\end{array}$ \\
\hline alk & $\begin{array}{r}0,001 \\
(0,001)\end{array}$ & $\begin{array}{c}0,005^{*} \\
(0,002)\end{array}$ & $\begin{array}{r}0,0004 \\
(0,001)\end{array}$ \\
\hline eat_h & $\begin{array}{r}0,0003 \\
(0,0004)\end{array}$ & $\begin{array}{r}0,0001 \\
(0,001)\end{array}$ & $\begin{array}{r}0,001 \\
(0,0005)\end{array}$ \\
\hline eat_reg & $\begin{array}{r}-0,002 * \\
(0,001)\end{array}$ & $\begin{array}{r}-0,001 \\
(0,001)\end{array}$ & $\begin{array}{r}-0,004^{*} \\
(0,001)\end{array}$ \\
\hline sport & $\begin{array}{c}-0,001 * \\
(0,0003)\end{array}$ & $\begin{array}{r}-0,001 * * * \\
(0,001)\end{array}$ & $\begin{array}{r}-0,002 * * * \\
(0,0004)\end{array}$ \\
\hline employment & $\begin{array}{c}0,004^{*} \\
(0,001)\end{array}$ & $\begin{array}{c}0,008^{*} \\
(0,002)\end{array}$ & $\begin{array}{c}0,003^{*} \\
(0,002)\end{array}$ \\
\hline wealth & $\begin{array}{r}0,0003 \\
(0,0003)\end{array}$ & $\begin{array}{r}0,0004 \\
(0,001)\end{array}$ & $\begin{array}{r}0,0003 \\
(0,0004)\end{array}$ \\
\hline year & $\begin{array}{c}-0,001 * \\
(0,0003)\end{array}$ & $\begin{array}{r}0,001 * * * \\
(0,0004)\end{array}$ & $\begin{array}{r}-0,003 * * * \\
(0,0003)\end{array}$ \\
\hline Константа & $\begin{array}{r}5,881^{*} \\
(0,508)\end{array}$ & $\begin{array}{r}1,65 * * \\
(0,794)\end{array}$ & $\begin{array}{r}8,23 * * \\
(0,661)\end{array}$ \\
\hline Наблюдения & 41720 & 16835 & 24885 \\
\hline R-sq within & 0,009 & 0,008 & 0,01 \\
\hline R-sq between & 0,206 & 0,142 & 0,272 \\
\hline R-sq overall & 0,181 & 0,12 & 0,236 \\
\hline Probability $(\mathrm{F})$ & 0,000 & 0,000 & 0,000 \\
\hline
\end{tabular}

Источник: Расчеты авторов.

Примечания: * - Значим на 1\%-ном уровне значимости; ** - значим на 5\%-ном уровне значимости; *** - значим на 10\%-ном уровне значимости. В скобках указаны стандартные очибки.

Относительно связи веса и демографических характеристик результаты Модели 1 подтвердили выводы дескриптивного анализа: и пол, и возраст выступают значимыми 
факторами величины ИМТ. Принадлежность к мужскому полу и молодой возраст при прочих равных условиях снижают ИМТ. Дополнительные годы жизни увеличивают вес, причем у женщин быстрее, чем у мужчин. Однако в пожилом возрасте ИМТ начинает понемногу снижаться: коэффициент при возрасте в квадрате значим и отрицателен. У мужчин этот процесс начинается уже с 62 лет, а у женщин - около 70 лет (о чем свидетельствуют соответствующие коэффициенты при переменных age/10 и age $2 / 100)$. Такой результат может объясняться, помимо прочего, эффектом самоотбора: в старшие возрастные группы переходят относительно здоровые респонденты, чей вес ниже, тогда как люди с морбидными формами ожирения умирают раньше.

Многие социально-экономические характеристики респондентов также оказались связанными с весом. Так, подтвердился вывод дескриптивного анализа о различной зависимости между весом и образованием у мужчин и женщин. И для той, и для другой группы среднее специальное и высшее образование оказалось значимым фактором, однако для мужчин более высокий уровень образования ассоциируется с высоким ИМТ, у женщин, наоборот, с низким.

Значимая положительная связь обнаружена между весом как мужчин, так и женщин, и наличием семьи и детей. Проживание в городе снижает вес при прочих равных условиях для представителей обеих гендерных групп. Проживание в столичном регионе увеличивает вес мужчин, но уменьшает вес женщин. И для мужчин, и для женщин характерна значимая положительная связь веса и трудовой занятости. Значимой связи веса и благосостояния обнаружить не удалось.

Определенные зависимости обнаружены между весом и факторами здорового/нездорового образа жизни. Так, регрессионный анализ подтвердил известную отрицательную зависимость полноты и факта курения как для мужчин, так и для женщин. У мужчин обнаружилась значимая положительная связь ИМТ и факта употребления алкоголя. Интенсивные занятия спортом, как и ожидалось, снижают вес, и эта зависимость также значима и для мужчин, и для женщин. Вопреки интуитивным ожиданиям, частота питания дома/вне дома оказалась незначимой переменной и для мужчин, и для женщин. Регулярность питания оказалась незначимым фактором формирования веса у мужчин. У женщин коэффициент при этой переменной значим, но его отрицательный знак также противоречит ожиданиям: те, кто питается регулярно, имеют более высокий вес при прочих равных условиях. К сожалению, обе переменные (питание дома и регулярность питания) не отражают его качества, а ведь питание может быть здоровым или нездоровым, независимо от места и частоты употребления пищи.

В целом результаты Модели 1 позволяют подтвердить гипотезы 1 u 2: избыточный вес связан с демографическими характеристиками индивидов, а также со многими социально-экономическими факторами и вредными/полезными привычками (хотя отдельные связи не всегда имеют место для обеих гендерных групп).

Для проверки гuпотезы 3 - связи избыточного веса и положения индивидов на рынке труда - использовались две регрессионные модели: вероятности занятости (Модель 2) и заработной платы (Модель 3), которые были представлены как функции от ряда переменных. Мы предполагаем, что и на вероятность занятости, и на уровень заработной 
платы оказывают влияние индивидуальные характеристики респондентов (пол, возраст, образование, семейный статус, ИМТ) и характеристики региональных рынков труда (город/село, столичный регион), а также макроэкономическая динамика (что можно контролировать переменными года наблюдения).

Вероятность занятости оценивалась на основе панельной выборки за 2012-2016 гг., в которую были включены индивиды от 19 до 72 лет (т.е. трудоспособные в соответствии с определением Международной организации труда, за исключением подростков 15-18 лет), ответившие на все вопросы, необходимые для формирования выбранных переменных. Выборка составила в итоге 33684 наблюдения по 12242 индивидам. Для проведения регрессионного анализа были сформированы следующие переменные.

Зависимая переменная:

- employment - занятость, принимает значение 1, если респондент работает или находится в отпуске; 0 - если не работает.

Независимые переменные:

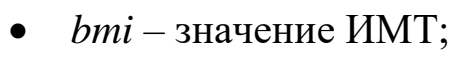

- $\quad b m i^{2}-$ ИМТ в квадрате;

- а также ряд независимых переменных, включавшихся выше в Модель 1: age/10, age2/100, sch1, sch2, sch3, marriage, city, capital, year.

Проверка выбранных независимых переменных на мультиколлинеарность не показала их тесной связи.

Оценивались пробит-модели вероятности занятости с фиксированными и случайными индивидуальными эффектами для всей выборки и отдельно для подвыборок мужчин и женщин. Все модели оказались статистически значимыми. Наилучшие результаты, с точки зрения значимости коэффициентов и тесноты связи, показали модели со случайными индивидуальными эффектами, они и были выбраны для анализа. Поскольку коэффициенты пробит-модели не могут интерпретироваться напрямую, были рассчитаны средние предельные эффекты, которые приведены в таблице 5 (Модель 2).

Результаты оценки показали, что интересующий нас коэффициент при переменной ИМТ значим как для всей выборки, так и для мужчин и женщин в отдельности, и имеет положительный знак. Это означает, что вероятность занятости возрастает одновременно с ростом веса респондента при прочих равных условиях. Коэффициент при переменной ИМТ в квадрате также значим и отрицателен, но по модулю он на порядок меньше, чем для ИМТ у мужчин, и на два порядка меньше, чем у женщин. Значит, только при очень высоких значениях ИМТ вероятность занятости начинает снижаться, причем очень медленными темпами. Мы видим также, что коэффициенты при контрольных переменных почти во всех случаях оказываются значимыми и по модулю большими, чем коэффициенты при ИМТ. Это значит, что вес работника не имеет такого большого значения для его занятости, как возраст, проживание в городе и образование. 
Таблица 5. Модель 2. Пробит-модель вероятности занятости: предельные эффекты

\begin{tabular}{|c|c|c|c|}
\hline & Вся выборка & Мужчины & Женщины \\
\hline \multicolumn{4}{|l|}{ Переменные: } \\
\hline male & $\begin{array}{c}0,069^{*} \\
(0,007)\end{array}$ & - & - \\
\hline$b m i$ & $\begin{array}{c}0,024 * \\
(0,004)\end{array}$ & $\begin{array}{c}0,042^{*} \\
(0,007)\end{array}$ & $\begin{array}{c}0,014 * \\
(0,005)\end{array}$ \\
\hline$b m i^{2}$ & $\begin{array}{r}-0,0004^{*} \\
(0,0001)\end{array}$ & $\begin{array}{l}-0,001 * \\
(0,0001)\end{array}$ & $\begin{array}{r}-0,0002 * * \\
(0,0001)\end{array}$ \\
\hline age/10 & $\begin{array}{c}0,629 * \\
(0,015)\end{array}$ & $\begin{array}{c}0,529 * \\
(0,022)\end{array}$ & $\begin{array}{r}0,664^{*} \\
(0,02)\end{array}$ \\
\hline $\operatorname{age}^{2} / 100$ & $\begin{array}{c}-0,081^{*} \\
(0,002)\end{array}$ & $\begin{array}{c}-0,072 * \\
(0,010)\end{array}$ & $\begin{array}{c}-0,085^{*} \\
(0,002)\end{array}$ \\
\hline \multicolumn{4}{|c|}{ Образование (база - незаконченное среднее): } \\
\hline $\operatorname{sch} 1$ & $\begin{array}{c}0,016 * \\
(0,007)\end{array}$ & $\begin{array}{r}0,025 * * \\
(0,012)\end{array}$ & $\begin{array}{r}0,007 \\
(0,011)\end{array}$ \\
\hline $\operatorname{sch} 2$ & $\begin{array}{r}0,1 * \\
(0,01)\end{array}$ & $\begin{array}{c}0,078^{*} \\
(0,013)\end{array}$ & $\begin{array}{c}0,119 * \\
(0,014)\end{array}$ \\
\hline $\operatorname{sch} 3$ & $\begin{array}{c}0,042 * \\
(0,006)\end{array}$ & $\begin{array}{c}0,126^{*} \\
(0,014)\end{array}$ & $\begin{array}{c}0,208 * \\
(0,014)\end{array}$ \\
\hline marriage & $\begin{array}{c}0,042 * \\
(0,006)\end{array}$ & $\begin{array}{c}0,169^{*} \\
(0,011)\end{array}$ & $\begin{array}{r}-0,018 * * \\
(0,008)\end{array}$ \\
\hline city & $\begin{array}{c}0,098 * \\
(0,007)\end{array}$ & $\begin{array}{c}0,107^{*} \\
(0,011)\end{array}$ & $\begin{array}{r}0,088^{*} \\
(0,01)\end{array}$ \\
\hline capital & $\begin{array}{r}0,015 \\
(0,008)\end{array}$ & $\begin{array}{r}0,028 * * \\
(0,013)\end{array}$ & $\begin{array}{r}0,005 \\
(0,01)\end{array}$ \\
\hline year & $\begin{array}{r}-0,002^{*} \\
(0,008)\end{array}$ & $\begin{array}{r}-0,001 \\
(0,002)\end{array}$ & $\begin{array}{r}-0,003 \\
(0,002)\end{array}$ \\
\hline Константа & $\begin{array}{c}3,887^{*} \\
(2,734)\end{array}$ & $\begin{array}{r}1,512 \\
(4,306)\end{array}$ & $\begin{array}{r}4,553 \\
(3,535)\end{array}$ \\
\hline Число наблюдений & 33684 & 14405 & 19579 \\
\hline R-sq within & 0,023 & 0,033 & 0,037 \\
\hline R-sq between & 0,31 & 0,409 & 0,391 \\
\hline R-sq overall & 0,263 & 0,324 & 0,336 \\
\hline Probability $(\mathrm{F})$ & 0,000 & 0,00 & 0,00 \\
\hline
\end{tabular}

Источник: Расчеты авторов.

Примечания: *-значим на 1\%-ном уровне значимости; **-значим на 5\%-ном уровне значимости; ***-значим на 10\%-ном уровне значимости. В скобках указаны стандартные ошибки.

Заработная плата оценивалась на основе панельной выборки за 2012-2016 гг., в которую были включены только работающие респонденты, включая тех, кто на момент опроса находился в отпуске, и ответившие на все необходимые вопросы для формирования выбранных переменных. Выборка составила в итоге 17692 наблюдения по 7489 индивидам.

Для проведения регрессионного анализа была сформирована зависимая переменная:

- $\ln (w)$ - логарифм заработной платы респондента в рублях (за месяц), проиндексированной по индексу потребительских цен (ИПЦ) к 2016 г.

В качестве независимых использовались все те же переменные, что и в модели вероятности занятости, в том числе ИМТ и ИМТ в квадрате. Проверка выбранных переменных на мультиколлинеарность не показала тесной связи.

Оценивались модели логарифма заработной платы с фиксированными и случайными индивидуальными эффектами для всей выборки и отдельно для подвыборок мужчин и 
женщин. Все модели оказались статистически значимыми. Как и в предыдущем случае, лучшие результаты с точки зрения значимости коэффициентов и тесноты связи продемонстрировали модели со случайными индивидуальными эффектами, которые и были выбраны для анализа. Результаты оценки моделей представлены в таблице 6 (Модель 3).

Таблица 6. Модель 3: зависимость между логарифмом заработной платы и ИМТ

\begin{tabular}{|c|c|c|c|}
\hline & Вся выборка & Мужчины & Женщины \\
\hline \multicolumn{4}{|l|}{ Переменные: } \\
\hline male & $\begin{array}{c}0,396^{*} \\
(0,012)\end{array}$ & - & - \\
\hline$b m i$ & $\begin{array}{c}0,019 * \\
(0,007)\end{array}$ & $\begin{array}{c}0,033^{*} \\
(0,012)\end{array}$ & $\begin{array}{r}0,005 \\
(0,009)\end{array}$ \\
\hline bmi2 & $\begin{array}{r}-0,0002 * * \\
(0,0001)\end{array}$ & $\begin{array}{r}-0,0004 * * \\
(0,0002)\end{array}$ & $\begin{array}{r}-0,0001 \\
(0,001)\end{array}$ \\
\hline age & $\begin{array}{c}0,466^{*} \\
(0,029)\end{array}$ & $\begin{array}{c}0,431 * \\
(0,041)\end{array}$ & $\begin{array}{c}0,474^{*} \\
(0,039)\end{array}$ \\
\hline age2 & $\begin{array}{r}-0,063^{*} \\
(0,003)\end{array}$ & $\begin{array}{r}-0,063^{*} \\
(0,005)\end{array}$ & $\begin{array}{c}-0,062^{*} \\
(0,004)\end{array}$ \\
\hline \multicolumn{4}{|c|}{ Образование (база - незаконченное среднее): } \\
\hline $\operatorname{schl}$ & $\begin{array}{r}0,015 \\
(0,013)\end{array}$ & $\begin{array}{r}-0,001 \\
(0,016)\end{array}$ & $\begin{array}{r}0,049 * * \\
(0,022)\end{array}$ \\
\hline $\operatorname{sch} 2$ & $\begin{array}{c}0,098^{*} \\
(0,016)\end{array}$ & $\begin{array}{c}0,093 * \\
(0,021)\end{array}$ & $\begin{array}{c}0,124 * \\
(0,024)\end{array}$ \\
\hline $\operatorname{sch} 3$ & $\begin{array}{c}0,332 * \\
(0,016)\end{array}$ & $\begin{array}{c}0,256^{*} \\
(0,023)\end{array}$ & $\begin{array}{c}0,401 * \\
(0,024)\end{array}$ \\
\hline marriage & $\begin{array}{r}0,018 * * \\
(0,011)\end{array}$ & $\begin{array}{c}0,102 * \\
(0,018)\end{array}$ & $\begin{array}{r}-0,016 \\
(0,014)\end{array}$ \\
\hline city & $\begin{array}{c}0,302^{*} \\
(0,013)\end{array}$ & $\begin{array}{c}0,304^{*} \\
(0,019)\end{array}$ & $\begin{array}{c}0,297 * \\
(0,018)\end{array}$ \\
\hline capital & $\begin{array}{c}0,605^{*} \\
(0,015)\end{array}$ & $\begin{array}{c}0,572 * \\
(0,021)\end{array}$ & $\begin{array}{r}0,632 * \\
(0,02)\end{array}$ \\
\hline year & $\begin{array}{c}0,012^{*} \\
(0,002)\end{array}$ & $\begin{array}{r}0,002 \\
(0,003)\end{array}$ & $\begin{array}{c}0,019^{*} \\
(0,003)\end{array}$ \\
\hline Константа & $\begin{array}{c}-15,29 * \\
(4,237)\end{array}$ & $\begin{array}{r}5,096 \\
(6,089)\end{array}$ & $\begin{array}{r}-30,27 * \\
(5,867)\end{array}$ \\
\hline Наблюдения & 17692 & 7922 & 9770 \\
\hline R-sq within & 0,005 & 0,002 & 0,012 \\
\hline R-sq between & 0,391 & 0,363 & 0,348 \\
\hline R-sq overall & 0,357 & 0,326 & 0,32 \\
\hline Probability $(\mathrm{F})$ & 0,000 & 0,000 & 0,000 \\
\hline
\end{tabular}

Источник: Расчеты авторов.

Примечания: * - Значим на 1\%-ном уровне значимости; ** - значим на 5\%-ном уровне значимости; *** - значим на 10\%-ном уровне значимости. В скобках указаны стандартные ошибки.

Коэффициенты при переменных ИМТ для мужчин оказались значимыми и положительными, при ИМТ в квадрате - значимыми и отрицательными. Таким образом, ИМТ и заработная плата в этой гендерной группе растут одновременно при небольших значениях ИМТ. Однако, начиная с определенного уровня ИМТ (с 41, т.е. при так называемых морбидных формах ожирения), его более высокие значения приводят к снижению заработной платы при прочих равных условиях. Для женщин вес оказывается статистически не связанным с заработной платой при прочих равных условиях. Как и в модели вероятности занятости, коэффициенты при ИМТ и ИМТ в квадрате по модулю меньше коэффициентов при контрольных переменных, которые тоже оказались 
значимыми. Таким образом, факторы возраста, образования, проживания в городе и, в особенности, проживания в столичном регионе в большей степени влияют на заработную плату и во многих случаях нивелируют слабое отрицательное влияние веса даже при высоких значениях ИМТ.

В целом результаты оценок Модели 2 и Модели 3 подтверждают гипотезу 3: наличие значимой связи индекса массы тела работника с вероятностью его занятости и (для мужчин) с размером заработной платы, но эта связь скорее положительная и не слишком сильная в сравнении с другими факторами.

Для тестирования гипотезы 4 - связи избыточного веса и индивидуальных затрат на лечение - оценивалась сумма расходов на лечение, оплаченных самим респондентом (или частные расходы). Она включала расходы на лекарства, амбулаторную помощь, лечение в больнице и лекарства в больнице за месяц в рублях, приведенные к 2016 г. по ИПЦ. Для проведения анализа в этом случае в панельную выборку 2012-2016 гг. вошли все респонденты в возрасте 19-100 лет, которые смогли ответить на необходимые вопросы. Количество наблюдений составило 17286, число респондентов - 8577.

Для проведения регрессионного анализа использовались: зависимая переменная Lncost - логарифм расходов на лечение за месяц в рублях в ценах 2016 г.; независимые переменные (аналогично Моделям 1-3) male, age/10, age $2 / 100$, wealth, sch1, sch2, sch3, marriage, city, capital, year.

Проверка выбранных переменных на мультиколлинеарность не показала тесной связи, что позволило включить их в модель одновременно. Модель 4 оценивалась для всей выборки, для гендерных групп, а также для трех «весовых» групп по отдельности. Во всех случаях оценивались модели с фиксированными и случайными индивидуальными эффектами, все они оказались статистически значимыми. Как и в случае Моделей 1-3, лучшее качество продемонстрировали модели со случайными эффектами, которые и были выбраны для анализа.

В таблице 7 представлены результаты оценивания Модели 4. Как видно, интересующий нас коэффициент при переменной ИМТ значим для всей выборки, а также в отдельности для женщин и для подвыборки людей, страдающих предожирением. Коэффициент во всех этих случаях положителен, что позволяет говорить о наличии определенной позитивной связи избыточного веса и затрат на лечение. Для мужчин, а также для людей с нормальным весом и ожирением, значимой связи веса и частных расходов на лечение не обнаруживается. Другие факторы (возраст, семейное положение, образование, место жительства) определяют медицинские расходы в большей мере, чем вес респондента. Таким образом, гипотеза 4 подтверждается частично.

Наконец, гипотеза 5 подтверждается результатами оценок всех приведенных моделей для женщин и мужчин по отдельности. Так, Модель 1 говорит о том, что принадлежность к мужскому полу отрицательно связана с весом; вес увеличивается с годами и для мужчин, и для женщин, затем снижается в конце жизни, однако у женщин этот процесс начинается значительно позже, чем у мужчин. Модель 1 подтверждает разнонаправленную связь образования и веса для мужчин и женщин, а также выявляет 
положительную связь веса с проживанием в столицах для мужчин и отрицательную - для женщин, положительную связь веса с потреблением алкоголя только для мужчин. Модель 2 показывает более сильную связь веса и вероятности занятости мужчин по сравнению с женщинами (коэффициенты при переменных ИМТ у мужчин по модулю значительно больше). Модель 3 подтверждает связь размера заработной платы и веса у мужчин, но среди женщин такой зависимости не выявляет. Наконец, Модель 4 подтверждает значимую положительную связь частных расходов на лечение и веса только для женщин, но не для мужчин.

Таблица 7. Модель 4: зависимость расходов на лечение и ИМТ

\begin{tabular}{|c|c|c|c|c|c|c|}
\hline & $\begin{array}{c}\text { Вся } \\
\text { выборка }\end{array}$ & Мужчины & Женщины & Норма & Предожирение & Ожирение \\
\hline \multicolumn{7}{|l|}{ Переменные: } \\
\hline male & $\begin{array}{l}-0,095^{*} \\
(0,023)\end{array}$ & - & - & $\begin{array}{r}-0,086^{* *} \\
(0,04)\end{array}$ & $\begin{array}{r}-0,077 * * \\
(0,036)\end{array}$ & $\begin{array}{r}-0,065 \\
(0,044)\end{array}$ \\
\hline$b m i$ & $\begin{array}{l}0,009^{*} \\
(0,002)\end{array}$ & $\begin{array}{r}0,006 \\
(0,004)\end{array}$ & $\begin{array}{l}0,011^{*} \\
(0,002)\end{array}$ & $\begin{array}{r}-0,003 \\
(0,01)\end{array}$ & $\begin{array}{r}0,022 * * \\
(0,01)\end{array}$ & $\begin{array}{r}0,005 \\
(0,004)\end{array}$ \\
\hline age/10 & $\begin{array}{l}-0,257^{*} \\
(0,036)\end{array}$ & $\begin{array}{l}-0,218^{*} \\
(0,069)\end{array}$ & $\begin{array}{l}-0,285^{*} \\
(0,043)\end{array}$ & $\begin{array}{r}-0,267 * \\
(0,057)\end{array}$ & $\begin{array}{r}-0,288^{*} \\
(0,06)\end{array}$ & $\begin{array}{r}-0,158 * * \\
(0,079)\end{array}$ \\
\hline $\operatorname{age}^{2} / 100$ & $\begin{array}{l}0,034^{*} \\
(0,003)\end{array}$ & $\begin{array}{l}0,032 * \\
(0,007)\end{array}$ & $\begin{array}{l}0,036^{*} \\
(0,004)\end{array}$ & $\begin{array}{l}0,035^{*} \\
(0,006)\end{array}$ & $\begin{array}{r}0,038^{*} \\
(0,006)\end{array}$ & $\begin{array}{l}0,025^{*} \\
(0,007)\end{array}$ \\
\hline wealth & $\begin{array}{r}0,013 * * \\
(0,006)\end{array}$ & $\begin{array}{r}0,028 * * \\
(0,012)\end{array}$ & $\begin{array}{r}0,007 \\
(0,007)\end{array}$ & $\begin{array}{r}0,024 * * \\
(0,012)\end{array}$ & $\begin{array}{r}0,022 * * \\
(0,01)\end{array}$ & $\begin{array}{r}0,001 \\
(0,01)\end{array}$ \\
\hline \multicolumn{7}{|c|}{ Образование (база - незаконченное среднее): } \\
\hline schl & $\begin{array}{r}0,017 \\
(0,031)\end{array}$ & $\begin{array}{r}0,014 \\
(0,053)\end{array}$ & $\begin{array}{r}0,016 \\
(0,039)\end{array}$ & $\begin{array}{r}0,022 \\
(0,057)\end{array}$ & $\begin{array}{r}0,052 \\
(0,051)\end{array}$ & $\begin{array}{r}-0,025 \\
(0,051)\end{array}$ \\
\hline $\operatorname{sch} 2$ & $\begin{array}{l}0,095 * \\
(0,032)\end{array}$ & $\begin{array}{r}0,115 * * * \\
(0,06)\end{array}$ & $\begin{array}{r}0,089 * * \\
(0,038)\end{array}$ & $\begin{array}{r}0,05 \\
(0,059)\end{array}$ & $\begin{array}{r}0,167 * \\
(0,05)\end{array}$ & $\begin{array}{r}0,069 \\
(0,051)\end{array}$ \\
\hline $\operatorname{sch} 3$ & $\begin{array}{r}0,21 * \\
(0,033)\end{array}$ & $\begin{array}{c}0,196^{*} \\
(0,058)\end{array}$ & $\begin{array}{r}0,213^{*} \\
(0,04)\end{array}$ & $\begin{array}{r}0,21 * \\
(0,059)\end{array}$ & $\begin{array}{l}0,266^{*} \\
(0,051)\end{array}$ & $\begin{array}{l}0,164 * \\
(0,056)\end{array}$ \\
\hline marriage & $\begin{array}{l}0,133^{*} \\
(0,022)\end{array}$ & $\begin{array}{r}0,171^{*} \\
(0,05)\end{array}$ & $\begin{array}{l}0,111^{*} \\
(0,025)\end{array}$ & $\begin{array}{l}0,159^{*} \\
(0,039)\end{array}$ & $\begin{array}{l}0,156^{*} \\
(0,036)\end{array}$ & $\begin{array}{r}0,086 * * \\
(0,037)\end{array}$ \\
\hline city & $\begin{array}{l}0,104 * \\
(0,023)\end{array}$ & $\begin{array}{l}0,139 * \\
(0,044)\end{array}$ & $\begin{array}{r}0,09 * \\
(0,028)\end{array}$ & $\begin{array}{l}0,172 * \\
(0,044)\end{array}$ & $\begin{array}{r}0,062 * * * \\
(0,037)\end{array}$ & $\begin{array}{l}0,095^{*} \\
(0,036)\end{array}$ \\
\hline capital & $\begin{array}{c}0,305^{*} \\
(0,025)\end{array}$ & $\begin{array}{l}0,334^{*} \\
(0,048)\end{array}$ & $\begin{array}{r}0,291 * \\
(0,03)\end{array}$ & $\begin{array}{l}0,382 * \\
(0,045)\end{array}$ & $\begin{array}{r}0,268^{*} \\
(0,04)\end{array}$ & $\begin{array}{l}0,286^{*} \\
(0,042)\end{array}$ \\
\hline year & $\begin{array}{r}0,007 \\
(0,007)\end{array}$ & $\begin{array}{r}0,015 \\
(0,013)\end{array}$ & $\begin{array}{r}0,005 \\
(0,008)\end{array}$ & $\begin{array}{r}0,003 \\
(0,014)\end{array}$ & $\begin{array}{r}0,003 \\
(0,011)\end{array}$ & $\begin{array}{r}0,012 \\
(0,011)\end{array}$ \\
\hline Константа & $\begin{array}{r}-8,154 \\
(13,446)\end{array}$ & $\begin{array}{r}-22,875 \\
(26,647)\end{array}$ & $\begin{array}{r}-3,464 \\
(15,589)\end{array}$ & $\begin{array}{r}-0,17 \\
(27,244)\end{array}$ & $\begin{array}{r}-0,128 \\
(22,671)\end{array}$ & $\begin{array}{r}-18,153 \\
(21,891)\end{array}$ \\
\hline Наблюдения & 17286 & 4864 & 12422 & 5093 & 6104 & 6089 \\
\hline R-sq within & 0,002 & 0,002 & 0,002 & 0,005 & 0,0001 & 0,004 \\
\hline R-sq between & 0,068 & 0,077 & 0,06 & 0,069 & 0,075 & 0,051 \\
\hline R-sq overall & 0,059 & 0,07 & 0,053 & 0,058 & 0,068 & 0,046 \\
\hline Probability $(\mathrm{F})$ & 0,000 & 0,000 & 0,000 & 0,000 & 0,000 & 0,000 \\
\hline
\end{tabular}

Источник: Расчеты авторов.

Примечания: *- Значим на 1\%-ном уровне значимости; **-значим на 5\%-ном уровне значимости; ***_значим на 10\%-ном уровне значимости. В скобках указаны стандартные ошибки.

\section{ЗАКЛЮЧЕНИЕ: ВЫВОДЫ И ОГРАНИЧЕНИЯ ИССЛЕДОВАНИЯ}

Проведенный анализ показал, что избыточный вес населения - проблема, характерная для России, как и для большинства развитых и развивающихся стран мира. Рост доли людей, 
страдающих ожирением, отчетливо наблюдается в течение всего последнего двадцатилетия, причем именно в последние годы темпы его увеличились. В 2016 г. более половины взрослого населения России старше 18 лет (62\%) страдало избыточным весом, в том числе $27 \%$-ожирением.

Как и во многих развитых странах [Sassi et al. 2009], ожирение в России более характерно для женщин, а предожирение - для мужчин. Однако за последние 20 лет ожирение среди мужчин росло ускоренными темпами, тогда как у женщин скорость его распространения была более умеренной, а предожирение стабилизировалось.

Обнаружены гендерные различия связи возраста с ожирением. Как и везде, в России показатели веса растут с возрастом. Но если мужчины быстро полнеют в молодости (от 15 до 40 лет), то у женщин рост избыточного веса ускоряется после 40 и особенно после 50 лет, когда его доминирующей формой становится ожирение.

Среднее специальное и высшее образование коррелируют с низким весом только у женщин. Для мужчин, напротив, более высокий уровень образования ассоциируется с высокими показателями предожирения и ожирения. Этот результат соотносится с выводами исследований по странам ОЭСР, которые также прослеживают однозначную связь образования и веса только для женщин, тогда как в отношении мужчин их результаты неочевидны [Sassi et al. 2009].

В числе социально-экономических факторов, положительно коррелирующих с весом и у мужчин, и у женщин, - наличие семьи и детей, трудовая занятость, проживание в сельской местности (что противоречит выводам некоторых исследований, связывающих ожирение и урбанизацию [Eid et al. 2008]). У мужчин наблюдается, однако, связь избыточного веса с фактом проживания в столичных регионах. Избыточный вес не снижается с ростом доходов (субъективным благосостоянием). Последнее обстоятельство ставит Россию в ряд стран, еще не достигших «порогового» уровня доходов, начиная с которого рост социально-экономического статуса (образования и материального благополучия) ассоциируется с низким весом [Sassi et al. 2009].

Показатели образа жизни также демонстрируют корреляцию с весом: вес положительно связан с употреблением алкоголя (у мужчин), отрицательно - с занятиями спортом, а также с курением (в обеих гендерных группах). Значимость образа жизни как одного из факторов ожирения подтверждает выводы многих зарубежных исследователей [Cohen-Cole, Fletcher 2008].

Оценки связи избыточного веса и положения работников на рынке труда, проведенные регрессионными методами, показали, что индекс массы тела является значимым фактором, коррелирующим с вероятностью занятости, а у мужчин - и с заработной платой. Однако в обоих случаях связь оказывается положительной для низких и средних значений ИМТ, и лишь в случаях тяжелого ожирения проявляется отрицательная зависимость. Кроме того, все прочие факторы, определяющие занятость и заработки (пол и возраст работника, его образование, проживание в городе и в столичном регионе) оказывают более существенное влияние на его положение на рынке труда, чем избыточный вес. Таким образом, мы пока явно не наблюдаем в России «штрафа» со стороны рынка труда 
для полных работников, который был обнаружен в ряде зарубежных исследований [Register, Williams 1990; Averett, Korenman 1996; Brown, Routon 2018]. Возможно, такой результат объясняется тем, что в России избыточный вес распространен давно и привычен, а темпы его роста пока не столь стремительны, как во многих других странах (рисунок 1).

Увеличение веса ассоциируется с ростом числа хронических заболеваний, ухудшением самооценки здоровья, растущей вероятностью инвалидности (эти результаты совпадают с полученными на более ранних данных РМЭЗ [Рощина, Гремченко 2016; Колосницына, Бердникова 2009]). Полные люди в среднем чаще попадают в больницу, покупают лекарства и тратят на них больше денег по сравнению с людьми с нормальным весом. Эти результаты соотносятся с выводами многих зарубежных исследований о более высоких медицинских расходах полных людей [Withrow, Alter 2011]. Однако эконометрический анализ подтвердил положительную связь веса и медицинских расходов только для женщин и для людей, страдающих предожирением, при прочих равных условиях.

В целом гипотезы эмпирического исследования подтвердились. Мы можем утверждать, что избыточный вес населения России связан с демографическими (пол, возраст) и социально-экономическими факторами, такими как образование, трудовая занятость, место жительства, семейное положение и наличие детей и др. ИМТ также коррелирован с показателями здорового/нездорового поведения населения. Определенную связь можно наблюдать между ИМТ и положением работника на рынке труда вероятностью занятости и уровнем заработной платы, между ИМТ и частными расходами на лечение. Кроме того, мы отмечаем существенные гендерные различия связей социальноэкономических факторов с избыточным весом.

В числе ограничений исследования надо отметить уже упомянутые выше свойства показателя ИМТ - он не всегда корректно отображает наличие избыточного веса, особенно в молодых возрастных категориях, где есть люди, серьезно занимающиеся спортом. Кроме того, значительное число респондентов не отвечают на вопрос о весе и/или росте, что не дает возможности определить для них показатель ИМТ и включить их в выборку для анализа. Некоторые респонденты просто не знают своего веса, потому что не следят за ним, другие не хотят его называть. Если предположить, что не называют вес те, кто стесняется своей полноты, то может оказаться, что наши оценки избыточного веса занижены. Точно так же могут оказаться заниженными и оценки последствий ожирения. Для решения этой проблемы нужны специальные регулярные эпидемиологические обследования, которые предусматривали бы обязательное измерение роста и веса респондента, а также дополнительные измерения для более корректного определения избыточного веса.

Кроме того, доступные данные РМЭЗ недостаточны для глубокого анализа связи веса и расходов на лечение, поскольку дают лишь оценки личных расходов индивидов, оставляя в стороне расходы государства.

Используемые в исследовании модели, как и любые эконометрические модели, не дают возможности выявить все факторы, связанные с избыточным весом, - часть из них неизбежно остаются ненаблюдаемыми. Вместе с тем используемая модель панельных 
данных с индивидуальными случайными эффектами позволяет частично решить эту проблему.

Кроме того, мы не оговаривали в этой работе вопросы возможной двусторонней зависимости избыточного веса и факта занятости и заработка индивида. Такие зависимости могут иметь место как в результате стрессов на работе, питания готовой едой, так и из-за относительного удешевления пищи при росте заработка. Для учета возможной эндогенности используются более сложные инструментальные методы, что может быть дальнейшим продолжением анализа и даст более точные результаты.

\section{ЛИТЕРАТУРА}

Колосницына М.Г, А.В. Бердникова (2009). Избыточный вес - сколько это стоит и что с этим делать? // Прикладная эконометрика. 3(15): 72-93.

Рощина Я.М., Е.П. Гремченко (2016). Факторы склонности к здоровому образу жизни // Вестник Российского мониторинга экономического положения и здоровья населения НИУ ВШЭ (RLMS-HSE). 6: 143-146.

Ásgeirsdóttir T. L., H. Corman, K. Noonan, N. Reichman (2015). Lifecycle effects of a recession on health behaviors: Boom, bust, and recovery in Iceland // NBER Working Papers. 20950.

Antonanzas F., R.Rodriguez (2010). Feeding the economics of obesity in the EU in a healthy way // European Journal of Health Economics. 11: 351-353.

Arena V.C., K.R. Padiyar, W.N. Burton, J.J. Schwerha (2006). The impact of body mass index on short-term disability in the workplace // Journal of Occupational and Environment Medicine. 48: 1118-1124.

Averett S., S. Korenman (1996). The Economic Reality of the Beauty Myth // The Journal of Human Resources. 31(2): 304-330.

Becker G., K. M. Murphy (1988). A theory of rational addiction // Journal of Political Economy. 96(4): 675-700.

Brown C., P.W. Routon (2018). On the distributional and evolutionary nature of the obesity wage penalty // Economics and Human Biology 28: 160-172.

Christakis N., J. Fowler (2007). The spread of obesity in a large social network over 32 years // The New England Journal of Medicine. 357: 370-379.

Cohen-Cole E., J.M. Fletcher (2008). Is Obesity Contagious? Social Networks vs. Environmental Factors in the Obesity Epidemics // Journal of Health Economics, 27(5): 1382-7.

Courtemanche C. (2009). Longer Hours and Larger Waistlines: The Relationship between Work Hours and Obesity // Forum for Health Economics and Policy. 12(2) (Article 2).

Courtemanche C., Carden A. (2011). Supersizing supercenters? The impact of Walmart Supercenters on body mass index and obesity // Journal of Urban Economics. 69: 165-181.

Eid J., H. Overman, D. Puga, M. Turner (2008). Fat city: Questioning the relationship between urban sprawl and obesity // Journal of Urban Economics. 63: 385-404.

Finkelstein E., C. Fiebelkorn, G. Wang (2005). The costs of obesity among full-time employees // American Journal of Health Promotion. 20: 45-51. 
Fogel R.W., Costa D.L. (1997). A theory of technophysio evolution, with some implications for forecasting population, health care costs, and pension costs. // Demography. 34. 1: 49-66.

Gardner G., B. Halweil (2000). Underfed and overfed: the global epidemic of malnutrition // Worldwatch. $150 \mathrm{p}$.

Geiker N.R.W., A. Astrup, M.F. Hjorth, A. Sjödin, L. Pijls, C. Rob Markus (2018). Does stress influence sleep patterns, food intake, weight gain, abdominal obesity, and weight loss interventions and vice versa? // Obesity Reviews. 19: 81-97.

Grossman M., E. Tekin, R.Wada (2014). Food prices and body fatness among youths // Economics and Human Biology. 12: 4-19.

Gupta N.G., J. Greve (2011). Overweight and obesity and the utilization of primary care physicians // Health Economics. 20 (S1): 53 - 67.

Komlos J., P. Smith, B. Bogin (2004). Obesity and the rate of time preference: is there a connection? // Journal of Biological Science. 36(2): 209-219.

Lallukka T., E. Lahelma, O. Rahkonen, E. Roos, E. Laaksonen, P. Martikainen, J. Head, E. Brunner, A Mosdol, M. Marmot, M. Sekine, A. Nasermoaddeli, S. Kagamimori (2008). Associations of job strain and working overtime with adverse health behaviors and obesity: Evidence from the Whitehall II Study, Helsinki Health Study, and the Japanese Civil Servants Study // Social Science \& Medicine. 66: 1681- 1698.

Levy E., P. Levy, C. Le Pen, A. Basdevant (1995). The economic cost of obesity: the French situation. // International Journal of Obesity Related Metabolic Disorders. 19: 788-792.

Lu Y., D. Goldman (2010). The Effects of Relative Food Prices on Obesity - Evidence from China: 1991-2006 // NBER Working Paper. 15720.

Morris S. (2007). The impact of obesity on employment // Labour Economics. 14: 413-433.

Park C., C. Kang (2008). Does education induce healthy lifestyle? // Journal of Health Economics. 27: 1516-1531.

Philipson T., R. Posner (2003). The long-run growth in obesity as a function of technological change // Perspectives in Biology and Medicine. 46(3) Supplement: 87-107.

Popkin B.M., S. Kim, E.R.S. Du Rusev, C. Zizza (2006). Measuring the full economic costs of diet, physical activity and obesity-related chronic diseases // Obesity Reviews. 7: 271-293.

Register Ch.A., D.R. Williams (1990). Wage Effects of Obesity among Young Workers // Social Science Quarterly. 71(1):130-41.

Ruhm C.J. (2005). Healthy living in hard times? // Journal of Health Economics. 24(2): 341363.

Rosin O. (2008). The Economic Causes of Obesity: A Survey // Journal of Economic Surveys. 22(4): 617-647.

Sargent J.D., D.G. Blanchflower (1994). Obesity and Stature in Adolescence and Earnings in Young Adulthood // Archives of Pediatrics and Adolescent Medicine. 148(7): 681-687.

Sassi F. (2010). Obesity and the Economics of Prevention: Fit Not Fat. Paris: OECD Publishing.

Sassi F., M. Devaux, M. Cecchini, E. Rusticelli (2009). The Obesity Epidemic: Analysis of Past and Projected Future Trends in Selected OECD Countries // OECD Health Working Papers. 45. Paris: OECD Publishing.

Stryjecki C., A. Alyass, D. Meyre (2018). Ethnic and population differences in the genetic predisposition to human obesity // Obesity Reviews. 19: 62-80. 
Stunkard A., J. Harris, N. Peersen, G. McClearn (1990). The body-mass index of twins who have been reared apart // New England Journal of Medicine. 322(21): 1483-1487.

Sturm R. (2002). The effects of obesity, smoking and drinking on medical problems and costs // Health Affairs (Millwood). 21: 245-253.

Tsai S.P., J.K. Wendt, F.S. Ahmed, R.P. Donnelly, T.R. Strawmyer (2005). Illness absence patterns among employees in a petrochemical facility: impact of selected health risk factors // Journal of Occupational and Environment Medicine. 47: 838-846.

Waaler H.Th. (1984). Height, weight and mortality: the Norwegian experience. Acta Medica Scandinavica. Supplement 679.

Withrow D., D.A. Alter (2011). The Economic Burden of Obesity Worldwide: A Systematic Review of the Direct Costs of Obesity // Obesity Reviews. 12(2): 131-141.

WHO (2017). Adolescent obesity and related behaviours: trends and inequalities in the WHO European Region, 2002-2014. WHO Regional Office for Europe: Copenhagen. 


\title{
OVERWEIGHT: SOCIOECONOMIC FACTORS AND CONSEQUENCES
}

\author{
MARINA KOLOSNITSYNA, OLGA KULIKOVA
}

\begin{abstract}
Increasing overweight is one of the leading global public health problems. In recent decades, this phenomenon has become a subject of intensive research in various fields of science. In Russia, the socioeconomic factors and consequences of overweight are insufficiently studied. This paper reviews the literature on the causes of overweight and the costs borne by individuals, employers, and the state. Based on microdata from the Russian Longitudinal Monitoring Survey for the years 2012-2016, we conduct an empirical analysis of obesity and pre-obesity in Russia. We investigate the links between overweight and demographic and socioeconomic factors, labour market outcomes and health spending.
\end{abstract}

Our results confirm an unambiguous connection between overweight and age, as well as gender of an individual. Overweight is also associated with unhealthy lifestyles. Among females, university education is correlated with low values of body mass index (BMI). Among males, on the contrary, a high level of education is associated with pre-obesity and obesity.

Regression estimates of overweight and labour outcomes show that BMI significantly correlates with employment probability and - for male workers - with wages. However, in both cases this correlation is positive for small or moderate BMI values, while for extreme obesity the correlation is negative.

\begin{abstract}
An increase in body weight is associated with an increasing number of chronic diseases, a worsening of selfrated health and a growing likelihood of disability. Compared to people with normal weight, obese people, on average, check into hospitals on a more frequent basis, buy pharmaceuticals more often and pay more for them. At the same time, econometric analysis confirms a positive correlation of weight and health spending only for women and people suffering from pre-obesity.
\end{abstract}

Key words: obesity, overweight, body mass index, obesity consequences, RLMS HSE, Russia.

MARINA Kolosnitsyna (mkolosnitsyna@hse.ru), NATIONAL RESEARCH UNIVERSity Higher SchOOL OF ECONOMICS, RUSSIA.

Olga Kulikova, National Research University Higher School of Economics, Russia.

THIS STUDY IS A PART OF THE PROJECT "HEALTH FORMATION: INPUT OF DIFFERENT FACTORS" FUNDED BY THE NATIONAL RESEARCH UNIVERSITY HighER SCHOOL OF ECONOMICS (GRANT N154, 2018).

DATE RECEIVED : SEPTEMBER 2018.

\section{REFERENCES}

Antonanzas F., R.Rodriguez (2010). Feeding the economics of obesity in the EU in a healthy way // European Journal of Health Economics. 11: 351-353.

Arena V.C., K.R. Padiyar, W.N. Burton, J.J. Schwerha (2006). The impact of body mass index on short-term disability in the workplace // Journal of Occupational and Environment Medicine. 48: 1118-1124.

Ásgeirsdóttir T. L., H. Corman, K. Noonan, N. Reichman (2015). Lifecycle effects of a recession on health behaviors: Boom, bust, and recovery in Iceland // NBER Working Papers. 20950.

Averett S., S. Korenman (1996). The economic reality of the beauty myth // The Journal of Human Resources. 31(2): 304-330. 
Brown C., P.W. Routon (2018). On the distributional and evolutionary nature of the obesity wage penalty // Economics and Human Biology 28: 160-172.

Christakis N., J. Fowler (2007). The spread of obesity in a large social network over 32 years // The New England Journal of Medicine. 357: 370-379.

Cohen-Cole E., J.M. Fletcher (2008). Is obesity contagious? Social networks vs. environmental factors in the obesity epidemics // Journal of Health Economics, 27(5): 1382-7.

Courtemanche C. (2009). Longer hours and larger waistlines: The relationship between work hours and obesity // Forum for Health Economics and Policy. 12(2) (Article 2).

Courtemanche C., Carden A. (2011). Supersizing supercenters? The impact of Walmart Supercenters on body mass index and obesity // Journal of Urban Economics. 69: 165-181.

Eid J., H. Overman, D. Puga, M. Turner (2008). Fat city: Questioning the relationship between urban sprawl and obesity // Journal of Urban Economics. 63: 385-404.

Finkelstein E., C. Fiebelkorn, G. Wang (2005). The costs of obesity among full-time employees // American Journal of Health Promotion. 20: 45-51.

Fogel R.W., Costa D.L. (1997). A theory of technophysio evolution, with some implications for forecasting population, health care costs, and pension costs. // Demography. 34. 1: 49-66.

Gardner G., B. Halweil (2000). Underfed and overfed: the global epidemic of malnutrition // Worldwatch. $150 \mathrm{p}$.

Geiker N.R.W., A. Astrup, M.F. Hjorth, A. Sjödin, L. Pijls, C. Rob Markus (2018). Does stress influence sleep patterns, food intake, weight gain, abdominal obesity, and weight loss interventions and vice versa? // Obesity Reviews. 19: 81-97.

Grossman M., E. Tekin, R.Wada (2014). Food prices and body fatness among youths // Economics and Human Biology. 12: 4-19.

Gupta N.G., J. Greve (2011). Overweight and obesity and the utilization of primary care physicians // Health Economics. 20 (S1): 53 - 67.

Kolosnitsyna M., A.Berdnikova (2009). Izbytochnyj ves - skol'ko ehto stoit i chto s ehtim delat'? [Overweight: What are its costs and what could be done?] // Prikladnaya ekonometrika [Applied Econometrics]. 3(15): 72-93.

Komlos J., P. Smith, B. Bogin (2004). Obesity and the rate of time preference: is there a connection? // Journal of Biological Science. 36(2): 209-219.

Lallukka T., E. Lahelma, O. Rahkonen, E. Roos, E. Laaksonen, P. Martikainen, J. Head, E. Brunner, A Mosdol, M. Marmot, M. Sekine, A. Nasermoaddeli, S. Kagamimori (2008). Associations of job strain and working overtime with adverse health behaviors and obesity: Evidence from the Whitehall II Study, Helsinki Health Study, and the Japanese Civil Servants Study // Social Science \& Medicine. 66: 1681- 1698.

Levy E., P. Levy, C. Le Pen, A. Basdevant (1995). The economic cost of obesity: the French situation. // International Journal of Obesity Related Metabolic Disorders. 19: 788-792.

Lu Y., D. Goldman (2010). The effects of relative food prices on obesity - Evidence from China: 1991-2006 // NBER Working Paper. 15720.

Morris S. (2007). The impact of obesity on employment // Labour Economics. 14: 413-433.

Park C., C. Kang (2008). Does education induce healthy lifestyle? // Journal of Health Economics. 27: 1516- 1531. 
Philipson T., R. Posner (2003). The long-run growth in obesity as a function of technological change // Perspectives in Biology and Medicine. 46(3) Supplement: 87-107.

Popkin B.M., S. Kim, E.R.S. Du Rusev, C. Zizza (2006). Measuring the full economic costs of diet, physical activity and obesity-related chronic diseases // Obesity Reviews. 7: 271-293.

Register Ch.A., D.R. Williams (1990). Wage effects of obesity among young workers // Social Science Quarterly. 71(1):130-41.

Roshchina Y.M., Y.P.Gremchenko (2016). Faktory sklonnosti k zdorovomu obrazu zhizni [Drivers of healthy lifestyle] // Vestnik Rossijskogo monitoringa ehkonomicheskogo polozheniya i zdorov'ya naseleniya NIU VSHE (RLMS-HSE) [Newsletter of the Russia Longitudinal Monitoring Survey NRU HSE (RLMS HSE)]. 6: 143-146.

Rosin O. (2008). The economic causes of obesity: A survey // Journal of Economic Surveys. 22(4): 617-647.

Ruhm C.J. (2005). Healthy living in hard times? // Journal of Health Economics. 24(2): 341363.

Sargent J.D., D.G. Blanchflower (1994). Obesity and stature in adolescence and earnings in young adulthood // Archives of Pediatrics and Adolescent Medicine. 148(7): 681-687.

Sassi F. (2010). Obesity and the Economics of Prevention: Fit Not Fat. Paris: OECD Publishing.

Sassi F., M. Devaux, M. Cecchini, E. Rusticelli (2009). The obesity epidemic: Analysis of past and projected future trends in selected OECD countries // OECD Health Working Papers. 45. Paris: OECD Publishing.

Stryjecki C., A. Alyass, D. Meyre (2018). Ethnic and population differences in the genetic predisposition to human obesity // Obesity Reviews. 19: 62-80.

Stunkard A., J. Harris, N. Peersen, G. McClearn (1990). The body-mass index of twins who have been reared apart // New England Journal of Medicine. 322(21): 1483-1487.

Sturm R. (2002). The effects of obesity, smoking and drinking on medical problems and costs // Health Affairs (Millwood). 21: 245-253.

Tsai S.P., J.K. Wendt, F.S. Ahmed, R.P. Donnelly, T.R. Strawmyer (2005). Illness absence patterns among employees in a petrochemical facility: impact of selected health risk factors // Journal of Occupational and Environment Medicine. 47: 838-846.

Waaler H.Th. (1984). Height, weight and mortality: The Norwegian experience. Acta Medica Scandinavica. Supplement 679.

WHO (2017). Adolescent obesity and related behaviours: Trends and inequalities in the WHO European Region, 2002-2014. WHO Regional Office for Europe: Copenhagen.

Withrow D., D.A. Alter (2011). The economic burden of obesity worldwide: A systematic review of the direct costs of obesity // Obesity Reviews. 12(2): 131-141.

Becker G., K. M. Murphy (1988). A theory of rational addiction // Journal of Political Economy. 96(4): $675-700$. 